\title{
Frequency shift, damping and tunneling current coupling with quartz tuning forks in non-contact atomic force microscopy
}

\author{
Laurent Nony ${ }^{1,2}$, Franck Bocquet ${ }^{1,2}$, Franck Para ${ }^{1,2}$, and Christian Loppacher ${ }^{1,2}$ \\ (1) Aix-Marseille Université, IM2NP, \\ Centre Scientifique de Saint-Jérôme, \\ Avenue Escadrille Normandie-Niemen, Case 151, \\ F-13397 Marseille CEDEX 20, France \\ ${ }^{(2)}$ CNRS, IM2NP (UMR 7334), Marseille-Toulon, France \\ Phys.Rev.B 94, 115421 (2016) \\ DOI: $10.1103 /$ PhysRevB.94.115421
}

(Dated: Received 1 March 2016; revised manuscript received 24 June 2016; published 16 September 2016)

\begin{abstract}
A combined experimental and theoretical approach to the coupling between frequency-shift $(\Delta f)$, damping and tunneling current $\left(I_{t}\right)$ in combined non-contact atomic force microscopy/scanning tunneling microscopy using quartz tuning forks (QTF)-based probes is reported. When brought into oscillating tunneling conditions, the tip located at the QTF prong's end radiates an electromagnetic field which couples to the QTF prong motion via its piezoelectric tensor and loads its electrodes by induction. Our approach explains how those $I_{t}$-related effects ultimately modify the $\Delta f$ and the damping measurements. This new paradigm to the origin of the coupling between $I_{t}$ and the nc-AFM regular signals relies on both, the intrinsic piezoelectric nature of the quartz constituting the QTF and its electrodes design.
\end{abstract}

PACS numbers: 41.20.Jb; 77.65.Fs; 77.65.-j; 68.37.Ef; 68.37.Ps

Keywords: non-contact Atomic Force Microscopy; Scanning Tunneling Microscopy; quartz tuning fork; frequency shift; tunneling current; piezoelectricity; electromagnetic coupling

\section{INTRODUCTION}

Quartz tuning forks (QTF) are used as oscillators in combined non-contact atomic force microscopy (ncAFM) and scanning tunneling microscopy (STM) since 2000 , with the pioneer work by F.Giessibl ${ }^{1}$ on $\mathrm{Si}(111) 7 \times$ 7. Their (i)-intrinsic piezoelectric properties, (ii)-thermal and spectral stability (resonance frequency $f_{0} \simeq 32 \mathrm{kHz}$, quality factor $Q \simeq 50000)$ and (iii)-large stiffness $(k \simeq$ $1800 \mathrm{~N} / \mathrm{m}$ ) render the probe highly sensitive to surface short-range (SR) atomic forces and hence, make QTF well-suited for scanning probe microscopy (SPM). These unique features, combined with the ability to control the tip apex with atomic precision at cryogenic temperatures, has triggered numerous outstanding results since $2009^{2-24}$

In 2011 however, discussions came up about QTFbased SPM probes. On $\mathrm{Si}(111) 7 \times 7$, several groups reported that there were a strong coupling between the tunneling current $I_{t}$ and the frequency-shift $(\Delta f)$ of the oscillating tip and hence, on the measured force $\mathrm{e}^{25,26}$. It is reminded that a major achievement of these probes is to allow the use of small oscillation amplitudes $\left(A_{0} \simeq 1 \AA\right)$, which makes the connection between $\Delta f$ and surface $\mathrm{SR}$ interaction forces straightforward ${ }^{27}$. To account for that $\Delta f / I_{t}$ coupling $^{28}$, two approaches were reported so far. A.Weymouth et al. evoked a repulsive phantom force on samples with limited conductivity such as semi-conductors $(\mathrm{SC})^{25,29}$. A year after, T.Wutscher et al. extended the frame of that model and proved that a metallic surface state did not prevent the phantom force from occurring ${ }^{30}$. This result went along with the work by M.Baykara et al. on the conductive oxidized $\mathrm{Cu}(100)$ surface, where a strong topography-feedbackinduced coupling on $\Delta f$ upon constant-current imaging was reported ${ }^{31}$. The second approach was introduced in 2012 by Z.Majzik et $a .^{26}$, who stated that part of the $\Delta f / I_{t}$ coupling stemmed from the electronics of the instrument (I/V converters, virtual ground issues...). Although refs. $[26,30]$ yet pointed out the electronic coupling was weak, a new design of probe was proposed wherein $I_{t}$ is collected by means of a thin insulated wire glued at the QTF prong's end ${ }^{26,29,32-34}$. This design was a valuable technical solution to lower the coupling and became a commercial standard, as well known as the qPlus sensor.

The above elements might bring to the conclusion that if the overall design of the QTF-based probe and its circuitry is optimized, the phantom force should couple $\Delta f$ and $I_{t}$ on SC samples, only. This article details that another coupling, which superposes to the "phantom effect" on SC, has to be accounted for during any QTF-based nc-AFM/STM experiment, including those on metallic substrates. When the tip oscillates in the vicinity of the surface in tunneling conditions, the AC part of the tunnelling current makes it behave as an antenna which radiates an electromagnetic (EM) field whose frequency is given by the oscillation frequency of the QTF. The EM radiated field is suspected to get coupled to the QTF, primarily by inverse piezoelectric effect. In this context, the coupling might intrinsically be related to the quartz material of the QTF and to its electrodes design. If at play, the coupling must alter the oscillation amplitude of the QTF detected by the nc-AFM electronics, which in turn must influence not only the frequency shift, but the damping as well. 


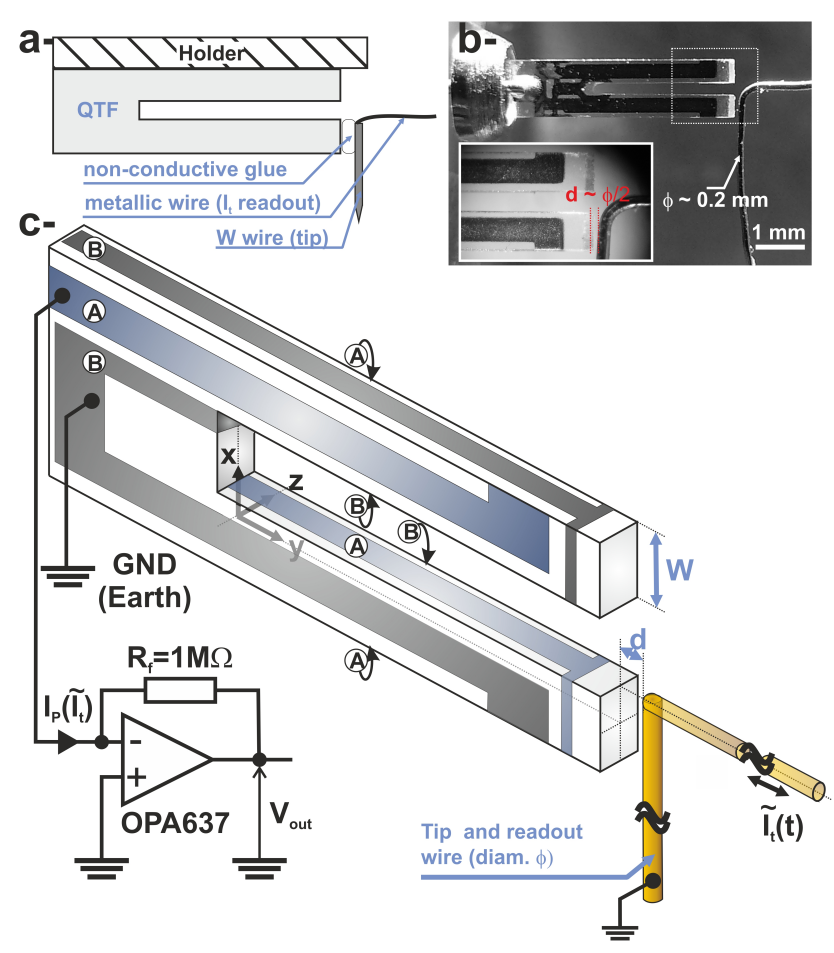

FIG. 1: (Color online) a-Scheme of a typical setup for a QTFbased SPM probe, after ${ }^{26,33}$, with one prong able to oscillate only. For the proper tunneling current readout, the tip is electrically decoupled from the QTF prong's end by an insulating medium. b-Picture of our experimental "proof of concept" setup supposed to mimic the setup schemed in a-. The elbow the wire forms is in the direct vicinity of one of the QTF's prongs. It is located at a distance $d \simeq \phi / 2 \simeq 0.1 \mathrm{~mm}$ from the QTF prong's end, however without touching it. c-Sketch of our setup including the geometry of an X-cut, $\alpha$-quartz, as used for QTF-based SPM probes. The electrodes' design is for illustrative purpose and does not depict the detailed trace of the actual electrodes of our QTF. The I/V converter is based after ${ }^{26}$.

In this work, the EM radiation-induced coupling with the QTF is evidenced. However, being concealed in the total piezoelectric current which is processed by the ncAFM electronics in a row, its consequences can hardly be traced with actual QTF-based SPM probes. Optical methods might be implemented to put them in evidence in-situ (UHV, low temperature), but this work seems difficult. We therefore built an otherwise simpler "proof of concept" setup which exemplifies the coupling.

The article is built as follows. In section II, the experimental setup and the results which testify the influence of the EM radiated field on the QTF are presented. The interpretation framework of those results, including a semi-quantitative description of the EM radiated field and of its coupling to the QTF is given in section III. At last, the consequences of our paradigm for the case of nc-AFM/STM combined experiments are discussed in section IV.

\section{EXPERIMENTAL SECTION}

\section{A. Setup}

The simplified sketch of a standard qPlus as reported in refs. [26,33] for instance and so-called "QTF-based SPM probe" in the following, is shown in fig.1a. The picture of our setup and the sketch of a QTF are reported in figs.1b and c, respectively. The aim of our setup is to put in evidence the EM radiation-induced coupling on a QTF by recording the subsequent piezoelectric current, $I_{p}$ (pA range), it develops.

For that purpose, $I_{p}(f)$ Fourier spectra (cf. subsection II C) are recorded as functions of the driving frequency $f_{d}$ and the magnitude $\widetilde{I}_{t}$ of the AC current flowing in a wire, $\widetilde{I}_{t}(t)=\widetilde{I}_{t} \cos \left(2 \pi f_{d} t\right)$ located near the QTF prong's end. We name that current $\widetilde{I}_{t}(t)$ for convenience because it is supposed to mimic the AC part of the tunneling current, as measured during a QTF-based SPM experiment. In our setup, we point out that the wire is never in contact with the QTF which is neither mechanically nor electrically driven (cf. fig.1c, B electrodes grounded). We are aware this does not mimic the detailed tip-wire geometry of an actual QTF-based SPM probe (gluing configuration, length and shape of the wire), but this is nevertheless suitable to evince the qualitative influence of the EM radiated field, as discussed in subsection III A.

A commercial QTF (LFXTAL002995 from IQD) was uncapped and fixed on a horizontal holder (not shown in fig.1b). It was placed at a distance $d$ from an elbowed $\mathrm{Cu}$ wire (diameter $\phi \simeq 0.2 \mathrm{~mm}$ for ease of manipulation) according to the geometry shown in fig.1b (here, $d \simeq$ $\phi / 2$ ). The wire is mounted on a slider (not shown in fig.1b), which permits to roughly adjust its distance $d$ to the QTF prong's end.

The AC current $\widetilde{I}_{t}(t)$ flowing in the wire is supplied by a function generator (Agilent 33120A) coupled to a $1 / 20$ voltage divider referenced to ground (Earth) and a $10 \mathrm{M} \Omega$ resistor (cf. fig.2). This allows us to achieve a range of current which is compatible with typical tunneling conditions during nc-AFM/STM combined experiments: $250 \mathrm{pA}$ to $1.25 \mathrm{nA}$ (peak values). The driving frequency $f_{d}$ may be tuned to match the resonance frequency of the anti-symmetric mode of the QTF, $f_{0}=32768 \mathrm{~Hz}$.

The piezoelectric current $I_{p}(t)$ supplied by the QTF is converted by an I/V stage with a basic design. Following the suggestions in ref.[26], we used as operational amplifier (OPA) an OPA637 from Texas Instruments (supply: $+/-15 \mathrm{~V})$ and a $R_{f}=1 \mathrm{M} \Omega$ feedback resistor. The 5Spice ${ }^{\complement}$ Analysis Pro software was used to verify the linearity of the I/V converter circuit. The simulated transfer function (TF) of the converter using the OPA637 SPICE model ${ }^{35}$ and a $R_{f}=1 \mathrm{M} \Omega$ feedback resistor is reported in fig.3a. An AC input current $I_{p}=10 \mathrm{pA}$ was used for the simulation. The shaded grey band depicts the $20-40 \mathrm{kHz}$ detection band. Thus, with these parameters in the $32 \mathrm{kHz}$ range, the OPA operates in its linear 


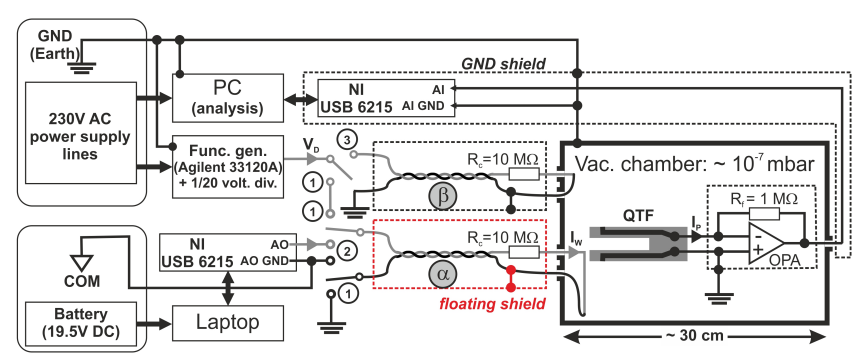

FIG. 2: (Color online) Scheme of the whole acquisition chain of the experimental setup shown in fig.1b. The QTF is placed in a vacuum chamber including the $\mathrm{I} / \mathrm{V}$ converter as well. Two wire configurations have been implemented to test the performances of the setup ( $\alpha$ and $\beta$ ), but the $\alpha$-one was used for the acquisition of the experimental spectra.

regime and does not act here as a charge amplifier ${ }^{26,36}$ Therefore $V_{\text {out }}(t)=-R_{f} I_{p}(t)$. As early mentioned, the only excitation source of the QTF is its thermal noise and the radiated EM field due to $\widetilde{I}_{t}(t)$, if any. In absence of radiated field $\left(\widetilde{I}_{t}=0\right)$, the total piezoelectric current $I_{p}(t)$ reduces to the current generated by thermal noise, $I_{\text {th }}$. This reference measurement is crucial as it gives access to the minimum perturbation detectable with our setup. The noise floor current $I_{\mathrm{nf}}$ is measured off resonance and includes $I_{\text {th }}$ (nearly null) and all noise sources issued from the circuitry.

The whole setup is placed within a vacuum chamber maintained at a pressure of about $10^{-7}$ mbar by means of an ionic pump in order to minimize mechanical vibrations. This allows for the investigation of the QTF under stable conditions with a well-pronounced resonance at $f_{0}=32768 \mathrm{~Hz}$ and a quality factor $Q \simeq 50000$. Measurements are performed at room temperature.

\section{B. Preventing ground coupling}

When measuring weak signals by means of active components such as OPAs, any external perturbation to the measurement, among which the ground coupling, is to be minimized. Therefore the design shown in fig. 2 takes great care to both, the shielding of the whole setup and the optimization of the ground connections. Along that line, note that the input (i.e. the wire) and output ( $V_{\text {out }}$ signal) feedthroughs are physically well-separated on the vacuum chamber and that the $\mathrm{I} / \mathrm{V}$ converter is located about $2 \mathrm{~cm}$ below the QTF within a grounded metallic box.

A more important issue deals with the stability of the virtual ground $\left(V_{\mathrm{GND}}\right)$ of the $\mathrm{I} / \mathrm{V}$ converter, i.e. the OPA's non-inverting input, which has to be continuously ensured. If $V_{\mathrm{GND}}$ is connected to ground and that the former fluctuates due to an external cause (current flow, external EM radiation...), this would immediately reverberate to the OPA output voltage. The strength of this effect depends on the choice and the regime of operation of the OPA, two aspects which have been discussed in ref.[26]. Albeit our OPA has good characteristics, one must make sure that the arbitrary connection of $V_{\mathrm{GND}}$ to the actual ground does not influence our measurement of $I_{p}(t)$. To carefully assess this, two strategies were used.

Firstly, we implemented two different wire configurations in the setup ( $\alpha$ and $\beta$, cf. fig.2). Compared to the $\alpha$ geometry described above, the $\beta$ configuration does not influence the QTF as it is located far away from it $(d>5 \mathrm{~cm})$. It is connected to ground and the AC current $\widetilde{I}_{t}(t)$ in it stems from the Agilent function generator (switch set to position 3 in fig.2). Since the QTF has no role in this configuration, if $\widetilde{I}_{t}(t)$ is to perturb the ground, this should readily be observed in $V_{\text {out }}(t)$ and hence in $I_{p}(f)$ Fourier spectra. Secondly, to get completely rid of the ground connection of the wire, we used a laptop supplied by a battery, i.e. with no electrical connection to the laboratory, and hence, no ground connection. A 16 bits, $250 \mathrm{kHz}$ board from National Instruments (NIUSB6215) connected to it was used as a digital to analog converter to provide the proper drive voltage to the $\alpha$ wire with a similar range of $\widetilde{I}_{t}(t)$ currents (switches set to position 2 in fig.2). In this configuration, the shield of the $\alpha$-wire is referenced to the COM point defined by the laptop battery.

Our observations are the following. When using the $\beta$-wire in the range of $\widetilde{I}_{t}(t)$ currents yet mentioned, no peak in the $I_{p}(f)$ Fourier spectrum was noticed, whatever the driving frequency that was used $\left(f_{d}=f_{0}\right.$ or $\left.f_{d} \neq f_{0}\right) . \quad I_{p}(f)$ remains constant and reaches the same noise floor as the one measured with the $\alpha$-wire (when $f_{d} \neq f_{0}$ ) in the ground-connected configuration, i.e. with the switches set to position 1. Note that this observation excludes any EM coupling to the ground as well. When exciting the $\alpha$-wire by means of the ground-free configuration (switches set to position 2), we get qualitatively similar results as when measuring with the groundconnected configuration. However, the ground-free configuration cannot be used for all experiments due to the limited duration of the laptop battery.

We therefore conclude the data presented hereafter acquired with the $\alpha$-wire in the ground-connected configuration are not altered by ground coupling.

\section{Data acquisition and post-processing}

The time-dependent piezoelectric current $I_{p}(t)$ is acquired by means of a 16 bits, $250 \mathrm{kHz}$ analog to digital converter (NI-USB6215) and a dedicated LabVIEW program. The data are acquired as follows: a buffer of $N=4250000$ samples of the signal $I_{p}(t)=-V_{\text {out }}(t) / R_{f}$ is acquired at $f_{s}=250 \mathrm{kHz}$. The windowing per buffer thus lasts $T_{\text {win }}=N / f_{s}=17 \mathrm{~s} . \quad I_{p}(t)$ is filtered by a $20 \mathrm{kHz}-40 \mathrm{kHz}$ 2nd order Butterworth bandpass filter implemented in the program to prevent aliasing from fre- 

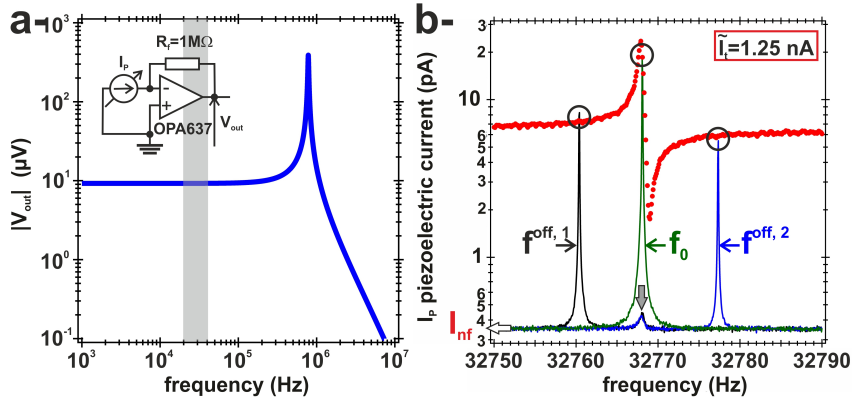

FIG. 3: (Color online). a-5Spice ${ }^{\circledR}$ simulation of the TF of the experimental I/V converter. The SPICE model of the OPA637 has been used. The input current magnitude (10 pA) is in the range of currents that are typically used during ncAFM/STM experiments. The shaded grey area depicts the $20-40 \mathrm{kHz}$ detection band. b-Illustrative sketch showing how $I_{p}^{\max }(f)$ spectra are derived (red-dotted curve). For each $\widetilde{I}_{t}$ value (here $\left.\widetilde{I}_{t}=1.25 \mathrm{nA}\right), I_{p}(f)$ averaged FFT spectra (log scale) are first recorded at one driving frequency: here for instance $f_{d}=f^{\text {off, } 1}=32760.4 \mathrm{~Hz}$ (black curve, 1024 spectra averaging), $f_{d}=f_{0}=32768 \mathrm{~Hz}$ (green curve, 1024 spectra averaging) or $f_{d}=f^{\text {off, }, 2}=32777.4 \mathrm{~Hz}$ (blue curve, 1024 spectra averaging). Note that upon those acquisition conditions, the noise floor current is well-visible $I_{\mathrm{nf}}=360 \mathrm{fA}$ and that when not driving at the resonance frequency, the thermal peak is visible (grey arrow) too. The value of the current corresponding to each driving frequency (maximum of the spectrum) is then stored (black circles) and the $I_{p}^{\max }(f)$ spectrum (red-dotted curve) is obtained upon sweep of $f_{d}$ over a given range.

quencies above the Nyquist frequency $f_{\mathrm{Nyq}}=f_{s} / 2=$ $125 \mathrm{kHz}$. The Fast Fourier Transform (FFT) spectrum of the buffer is then computed. The spectral resolution is $\delta f_{s}=f_{s} / N \simeq 60 \mathrm{mHz}$, which ensures the proper resolution of the QTF resonance peak whose full width at half maximum is $\Delta f_{\mathrm{FWHM}}=f_{0} / Q \lesssim 1 \mathrm{~Hz}$. The experimental observables are the modulus of the normalized monolateral FFT spectrum of $I_{p}(t), I_{p}(f)$ (in pA), and the rms value of the Power Spectrum Density (PSD) of $I_{p}(t): S(f)$ (in $\mathrm{pA} / \sqrt{\mathrm{Hz}}$ ). The two former quantities are derived from $I_{p}(t)$ according to:

$$
\left\{\begin{array}{l}
I_{p}(f)=\frac{2}{N}\left|\operatorname{FFT}\left\{I_{p}(t)\right\}\right| \\
S(f)=I_{p}(f) \sqrt{T_{\text {win }}}
\end{array}\right.
$$

FFT spectra are averaged to optimize the signal to noise ratio. The number of averaged spectra $\left(N_{\mathrm{av}}\right)$ for each acquisition is specified in each figure caption, but typically $N_{\text {av }}>1000$, which makes the acquisition duration of a single averaged spectrum last $\simeq 5 \mathrm{~h}$.

This is exemplified in fig.3b, where $I_{p}(f)$ averaged FFT spectra $\left(N_{\mathrm{av}}=1024\right)$ are reported for $\widetilde{I}_{t}=1.25 \mathrm{nA}$ and three driving frequencies: the resonance frequency of the QTF, $f_{d}=f_{0}=32768 \mathrm{~Hz}$ (green curve), and two offresonance frequencies, namely $f_{d}=f^{\text {off, } 1}=32760.4 \mathrm{~Hz}$

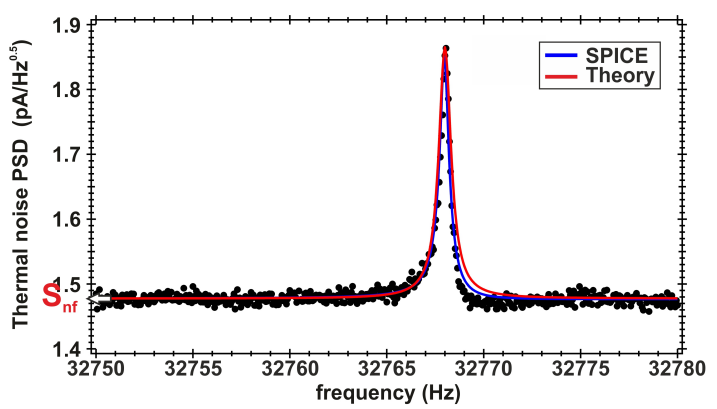

FIG. 4: (Color online). Thermal noise PSD of the QTF (black dots, 15530 spectra averaging). The experimental curve is fitted with the theoretical PSD of a 1D free harmonic oscillator (red curve), giving the equivalent mechanical parameters of the fork, namely $f_{0}=32768 \mathrm{~Hz}, Q=45000$ and $k=2700 \mathrm{nN} / \mathrm{nA}$. The blue curve is the 5 Spice $^{(}$thermal noise TF of the equivalent RLC circuit of a QTF. The derived equivalent electrical parameters are: $R=70 \mathrm{k} \Omega, L=24750 \mathrm{H}$, $C=953.16 \mathrm{aF}$.

and $f_{d}=f^{\text {off, } 2}=32777.4 \mathrm{~Hz}$ (black and blue curves respectively). Each spectrum exhibits a net peak at its driving frequency. When $f_{d} \neq f_{0}$, the magnitude of the peaks $(\simeq 7 \mathrm{pA})$ overcomes the magnitude of the thermal peak by a factor of $70\left(I_{\text {therm }} \simeq 90 \mathrm{fA}\right)$, which remains visible at $f_{0}$ otherwise (grey arrow). When $f_{d}=f_{0}$, the magnitude of the peak reaches $\simeq 20 \mathrm{pA}$, which therefore cannot be explained by the superposition of the offresonance current peaks and the thermal peak. Besides, the noise floor current (cf. white arrow) is consistent with the situation $\widetilde{I}_{t}=0$.

In the following however, those averaged FFT spectra are not reported as is, because each of them is the trace of only one drive frequency. The spectral representation we rather adopted is built as follows. Instead of averaging $I_{p}(f)$ over a large set of spectra at constant $f_{d}$, we record less spectra $(\lesssim 10)$ and track the magnitude of the $f_{d^{-}}$ peak, $I_{p}^{\max }\left(f=f_{d}\right)$, while sweeping $f_{d}$ over a frequency domain. The resulting $I_{p}^{\max }(f)$ spectrum is therefore similar to the transfer function (TF) of the QTF upon excitation by the radiated EM field, a spectrum not measurable otherwise. In fig.3b, the red-dotted curve is the asderived $I_{p}^{\max }(f)$ spectrum of the QTF for $\widetilde{I}_{t}=1.25 \mathrm{nA}$. The black circles exemplify how the spectrum was derived. The acquisition of $I_{p}^{\max }(f)$ spectra is long too, and the signal-to-noise ratio less good than for longlasting averaged spectra. The use of a standard Gaussian smooth procedure is useful then. All $I_{p}^{\max }(f)$ spectra reported hereafter were consistently recorded with the parameters: $f_{d}$-range $=32745-32795 \mathrm{~Hz}, 1000$ points (spectral resolution: $50 \mathrm{mHz}$ ); 15 averaged spectra per $f_{d}$-point; Gaussian smooth $=3$ points. At last, note that in the following, we will rather use the name of " $I_{p}(f)$ spectra" instead of the one of " $I_{p}^{\max }(f)$ spectra". 

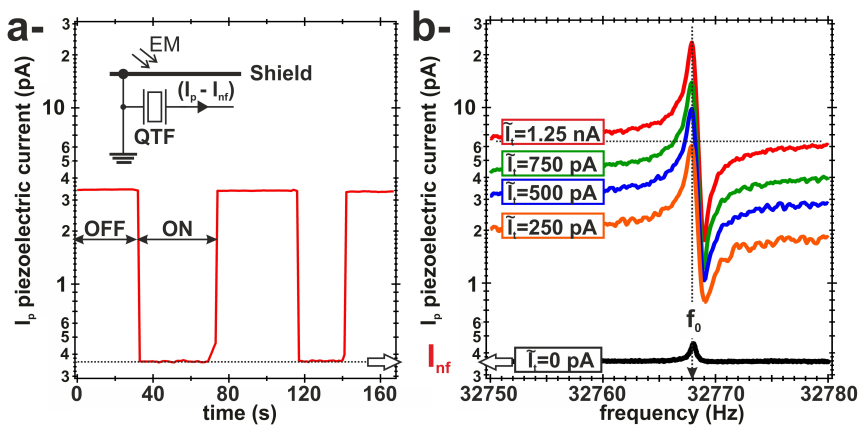

FIG. 5: (Color online). a-Piezoelectric current (log scale) vs. time recorded upon placing a grounded shield between the radiating wire and the QTF. Current in the wire: $250 \mathrm{pA}$, $f_{d}=32752 \mathrm{~Hz}$. The presence of the shield in front of the QTF makes the piezoelectric current dropping down to nearly the noise floor current, which testifies the influence of the radiated EM field on the QTF. b- $I_{p}(f)$ spectra for various $\widetilde{I}_{t}$ currents.

\section{Results}

\section{Thermal response of the QTF $\left(\widetilde{I}_{t}=0\right)$}

The noise floor current, $I_{\mathrm{nf}}$, of our detection setup as well as the QTF mechanical parameters are first determined. These are extracted from the thermal noise spectrum of the QTF, $I_{\mathrm{th}}$. The thermal noise Power Spectral Density (PSD) of the QTF without radiated field $\left(\widetilde{I}_{t}=0\right)$ is reported in fig.4. The spectrum is fitted with the thermal noise PSD of a 1D-free oscillator (red curve, Eq.3 in ref.[37]) within the $32750-32780 \mathrm{~Hz}$ range, giving the noise floor PSD $S_{\mathrm{nf}} \simeq 1.48 \mathrm{pA} / \sqrt{\mathrm{Hz}}$ (white arrow), a corresponding noise floor current $I_{\mathrm{nf}} \simeq 360 \mathrm{fA}$ and a thermal noise current at resonance $I_{\mathrm{th}}\left(f_{0}\right) \simeq 90 \mathrm{fA}$ above $I_{\mathrm{nf}}$. The integrated noise at $f_{0}$ is $\simeq 150 \mathrm{pA}$, hence the necessity to average our spectra. The other fitted parameters are the QTF's resonance frequency, quality factor and stiffness: $f_{0}=32768 \mathrm{~Hz}, Q=45000$ and $k \simeq 2700 \mathrm{nN} / \mathrm{nA}$.

The analysis is completed by the 5 Spice ${ }^{\complement}$ simulation of the thermal noise TF of the equivalent RLC circuit of the QTF (cf. blue curve in fig.4 and subsection III C). An offset of $1.34 \mathrm{pA} / \sqrt{\mathrm{Hz}}$ has been applied to the 5 Spice ${ }^{(}$ simulation to make it match the experimental noise floor. We believe this offset stems from our circuitry (cabling, local resistances,...). The asymmetry seen in the experimental spectrum, partly reproduced by the simulation, is attributed to OPA non-linearities when operating at that low currents (similar order of magnitude between $I_{p}$ and the OPA's input bias current). When operating at higher currents, the asymmetry disappears. The optimum electrical parameters we have found are: $R=70 \mathrm{k} \Omega$, $L=24750 \mathrm{H}, C=953.16 \mathrm{aF}$, in agreement with typical values for standard $\mathrm{QTF}^{38}$.

\section{Response of the QTF to an EM radiated field $\left(\widetilde{I}_{t} \neq 0\right)$}

To evidence the influence of the EM radiated field on the QTF, we first made a simple shielding experiment. If the EM field stemming from the wire is to influence the QTF, then placing a grounded metallic shield between the wire and the QTF must strongly attenuate the coupling. For ease of manipulation (placing/removing the shield), the experiment was performed in air with the wire placed at about $1 \mathrm{~mm}$ from the QTF prong's end. The QTF resonance being strongly attenuated in air, we used an arbitrary off-resonance driving frequency $f_{d}=32752 \mathrm{~Hz}$ and $\widetilde{I}_{t}=250 \mathrm{pA}$. Thus, a grounded molybdenum plate was manually and sequentially placed (ON state) and removed (OFF state) between the wire and the QTF with the resulting piezoelectric current $I_{p}(t)$ shown in fig.5a. In the ON state, the piezoelectric current vanishes down to the noise floor current $I_{\mathrm{nf}}=360 \mathrm{fA}$.

We now investigate the influence of the coupling in standard conditions (vacuum, $\alpha$-configuration and $d \simeq$ $100 \mu \mathrm{m}$ ), as defined before (cf. fig.1b). $I_{p}(f)$ experimental spectra (log scale) are reported in fig.5b. They were recorded for $\widetilde{I}_{t}=1.25 \mathrm{nA}, 750 \mathrm{pA}, 500 \mathrm{pA}$ and $250 \mathrm{pA}$ (peak values). The thermal noise spectrum $\left(\widetilde{I}_{t}=0\right)$ of fig. 4 has been reported as a reference curve too. The experimental spectra exhibit a resonance $\left(f_{0}=\right.$ $32768 \mathrm{~Hz})$ /anti-resonance $(32769 \mathrm{~Hz})$ behavior superimposed to a nearly constant background. The magnitude of both, resonance/anti-resonance currents and the background current increases with $\widetilde{I}_{t}$. At first glance, the background-shift and the current at resonance scale linearly with $\widetilde{I}_{t}$. This feature will be discussed in the following section and more specifically in subsection IIID.

Both, the shield experiment and the peculiar shape of the spectra unambiguously reveal the interaction between the EM radiation field and the QTF. The resonance/anti-resonance behavior needs to be interpreted, which can only be performed upon estimation of the EM radiation field and its coupling to the QTF.

\section{INTERPRETATION FRAMEWORK}

In order to interpret the experimental spectra and establish their relevance in the case of actual QTF-based SPM probes, the geometry of the probe sketched in figs.6a and $\mathrm{c}$ is rationalized and compared to the one of our setup (cf. fig.6b, reported from fig.1b): (i)-the tip wire is described by a cylindrical perfect conductor with a diameter $\phi$ and a length $L$. It is vertically oriented; (ii)-the axis of the cylinder is parallel to the end face of the QTF and located at a distance $d \simeq \phi / 2$ from it. Hence, $d$ stands for the glue thickness in the actual probe; (iii)-the length of the tip is much larger than the thickness of the QTF prong $W, L \gg W$; (iv)-the tunneling current read-out wire is described with the same kind of cylinder as the tip, except that it is horizontal and has 
a semi-infinite length; (v)-the elbowed-junction between the tip and the read-out wire is described by a quarter of circle whose radius $R_{w} \simeq \phi$.

In our setup, the tip wire is long and does not end at the location where the tunnel junction might be (cf. dotted line in fig.6c). Its return path to ground forms another elbow (sketched in fig.6b) which is located several millimeters away from the QTF. Hence, the overall shape of the wire compares to a large current loop. This geometry obviously differs from the design of an actual probe. But, subsection III A (Eq.2) will establish that the $\mathrm{AC}$ current flowing in the wire may be interpreted as a superposition of infinitesimal oscillating dipoles located along it. On the other hand, the occurrence of a tunneling current requires the use of a semi-infinite grounded metallic substrate. Thus, the AC current in the wire has to be completed by its corresponding set of image dipoles in the metal body in order to ensure the zero potential of the surface which therefore acts as an anti-symmetrical plane in the electromagnetic problem.

In this description, the precise influence of the tunnel junction, which obviously depends on the exact tip apex geometry, is not accounted for. However, we infer it behaves like a infinitesimal dipolar contribution as well, with a dipole magnitude proportional to $\widetilde{I}_{t} d_{j} /\left(2 \pi f_{d}\right)$ (cf. subsection III A), $d_{j}$ being the tip/surface separation. For $L \gg d_{j}$, the influence of the resulting "junction dipole" will therefore not weight predominantly in the resulting EM radiation field. Thus, by dint of image effects, this situation is also similar to a "loop" of current, which is precisely the case mimicked in our setup.

We hereafter detail the derivation of the EM field radiated by a loop of current whose simplified geometry is built as follows (cf. fig.6c): (i)-the tip wire of length $L$ and its corresponding set of image dipoles, i.e. a segment of length $2 L$ ( $A^{\prime} A$ segment); (ii)-the elbowed area ( $A B$ curve) and its image counterpart $\left(A^{\prime} B^{\prime}\right)$; (iii)-the readout wire depicted as a semi-infinite connection to the I/V converter $\left(B C\right.$ segment) with its images too $\left(B^{\prime} C^{\prime}\right.$ segment).

\section{A. Description of the EM radiated field}

Solving exactly the electromagnetic problem including a current source facing a QTF (dielectric medium + metallic electrodes) with the proper boundary conditions in an analytical way seems impossible. Therefore, despite sketched in fig.6c for illustrative purpose, the QTF is not taken into consideration in the way we solve the electromagnetic problem. We only solve the problem for an isolated tip in vacuum, with the simplified geometry yet described.

As stated by standard antenna theory ${ }^{39}$, for a wire with a high aspect-ratio (length/radius) and a negligible spatial extension w.r.t. the corresponding current wavelength $\lambda$, the instantaneous charge distribution due to an AC current $\widetilde{I}_{t}(t)=\widetilde{I}_{t} \cos (2 \pi f t)$ is approximated by its

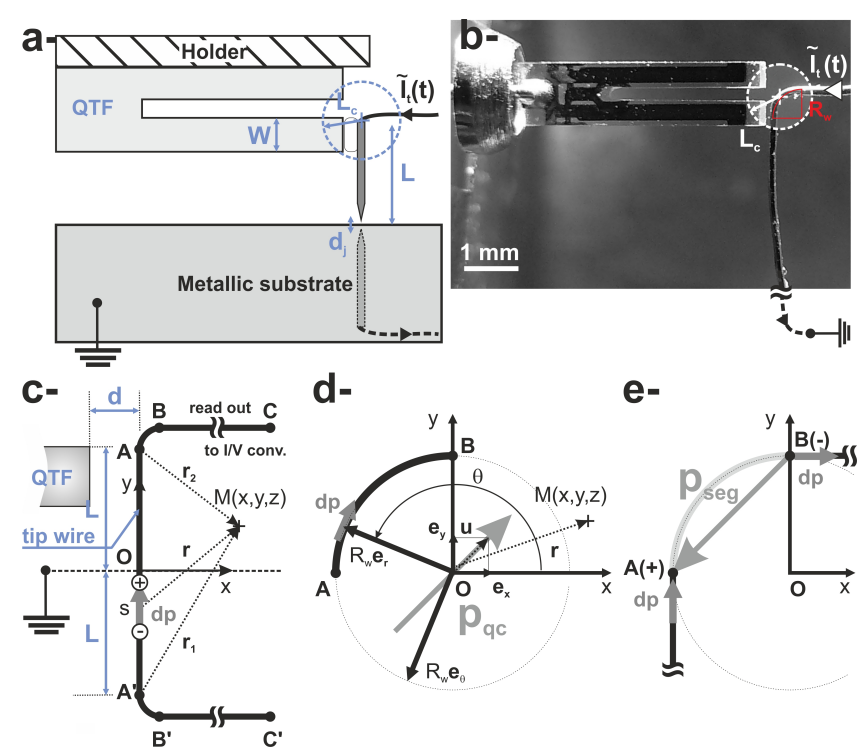

FIG. 6: (Color online) a-Simplified geometry of an actual QTF-based SPM probe, as shown in fig.1a. The dotted circle (radius $L_{c}$ ) depicts the elbowed area of the wire where the major part of the EM radiated field able to influence the QTF will stem from. The metallic substrate's surface (grounded) acts as an anti-symmetrical plane in the electromagnetic problem (image dipoles effects). b-Picture of the geometry of our "proof of concept" setup. The return path to ground is located far away from the QTF, consistently with the situation in a- when image dipoles effects are considered. c-Sketch of the geometry used for the derivation of the EM radiated field. Three distinct zones and their corresponding images are considered, which can be summarized as: the $A A^{\prime}$ segment of length $2 L$; the $B C$ and $B^{\prime} C^{\prime}$ semi-infinite segments; the $A B$ and $A^{\prime} B^{\prime}$ curves. The EM radiated may be estimated for each of them (see text). d-Detailed geometry of the $A B$ curve (elbowed area) used for the derivation of the EM radiated field. That part is equivalent to a dipolar contribution $\mathbf{p}_{\mathrm{qc}}$. e-Detailed geometry of the $A$ - and $B$-points belonging to the segments regions which are involved in the EM radiated field able to influence the most the QTF. Eq.4 states that $A-(B-)$ point is positively (negatively) charged, hence defining a dipole $\mathbf{p}_{\text {seg }}$.

first non-vanishing multipolar expansion term, i.e. the dipolar one. Each infinitesimal part of the wire with a length $d s \ll r(r$ being the distance at which this part is observed) exhibits a dipolar moment $\mathbf{d} \mathbf{p}(t)=\widetilde{I}_{t}(t) \mathbf{d} \mathbf{s} / \omega$, where $\omega=2 \pi f$. In the proximity zone $r \ll \lambda=c / f$ for which retardation effects can be ignored, the radiated EM field (magnetic and electric components $\mathbf{B}_{\mathrm{rad}}$ and $\mathbf{E}_{\text {rad }}$, respectively) has mainly an electric character: $B_{\mathrm{rad}} / E_{\mathrm{rad}} \simeq c \epsilon_{0} r / \lambda \ll 1$, where $c$ is the light celerity and $\epsilon_{0}$, the dielectric permittivity of vacuum. Using standard complex notations $\widetilde{I}_{t}(t)=\widetilde{I}_{t} e^{-j \omega t}$, where only the real part has physical relevance, the electric radiated field $\mathbf{d E}_{\text {rad }}(\mathbf{r}, t)$ produced by an infinitesimal dipole $\mathbf{d p}(t)$ is given by: 


$$
\mathbf{d E}_{\mathrm{rad}}(\mathbf{r}, t)=\frac{1}{4 \pi \epsilon_{0}} \frac{j \widetilde{I}_{t}}{\omega} e^{-j \omega t}\left(\frac{3 \mathbf{r} \cdot \mathbf{d} \mathbf{s}}{r^{5}} \mathbf{r}-\frac{\mathbf{d s}}{r^{3}}\right),
$$

where $\mathbf{r}$ is the vector joining the dipole "center" to the observation point (cf. fig.6c). In the proximity zone approximation, the EM radiated field is then obtained by integrating $\mathbf{d E}_{\mathrm{rad}}$ along the curvilinear abscise following the wire shape.

\section{Radiated EM field by a straight segment of length $2 L$}

Following the geometry of fig.6c, the radiated field by the $A A^{\prime}$ segment is:

$$
\begin{aligned}
\mathbf{E}_{\mathrm{rad}}^{\mathrm{seg}}(M, t) & =\int_{-L}^{L} \mathbf{d} \mathbf{E}_{\mathrm{rad}}(\mathbf{r}, t) d s \\
& =\frac{1}{4 \pi \epsilon_{0}} \frac{j \widetilde{I}_{t}}{\omega} e^{-j \omega t}\left\{\begin{array}{l}
\int_{-L}^{L} \frac{3 x(y-s)}{r_{s}^{5}} d s \\
\int_{-L}^{L}\left[\frac{3(y-s)^{2}}{r_{s}^{5}}-\frac{1}{r_{s}^{3}}\right] d s \\
\int_{-L}^{L} \frac{3 z(y-s)}{r_{s}^{5}} d s
\end{array}\right.
\end{aligned}
$$

where $r_{s}=\|\mathbf{r}\|=\sqrt{x^{2}+(y-s)^{2}+z^{2}}$. The equation may be recast into:

$$
\mathbf{E}_{\mathrm{rad}}^{\mathrm{seg}}(M, t)=\frac{1}{4 \pi \epsilon_{0}} \frac{j \widetilde{I}_{t}}{\omega} e^{-j \omega t}\left[\frac{\mathbf{r}_{\mathbf{2}}}{r_{2}^{3}}-\frac{\mathbf{r}_{\mathbf{1}}}{r_{1}^{3}}\right],
$$

$\mathbf{r}_{1}$ and $\mathbf{r}_{2}$ being the vectors joining the beginning and the end of the segment to the $\mathrm{M}$ point, respectively. For $A A^{\prime}$ segment, $\mathbf{r}_{\mathbf{2}}=\mathbf{r}_{\mathbf{1}}-2 L \mathbf{e}_{\mathbf{y}}$, and Eq.4 stands for the field created by a negative (positive) charge in $A^{\prime}(A)$.

\section{Radiated EM field by a quarter of circle}

The current is supposed to flow through a quarter of circle belonging to the $(x, y)$ plane with the geometry shown in fig.6d (curve $A B$, flowing orientation from $A$ to $B$ here $)$. The observation point $\mathrm{M}(\mathrm{x}, \mathrm{y}, \mathrm{z})$ is referenced to the center of the circle by the vector $\mathbf{r}$. The elementary dipole is given in standard polar coordinates $\left(\mathbf{e}_{\mathbf{r}}, \mathbf{e}_{\theta}\right)$ by $\mathbf{d p}=\widetilde{I}_{t} R_{w} d \theta \mathbf{e}_{\theta} / \omega$, where $R_{w}$ is the radius of the circle and $d \theta$ the elementary polar angle.

The field in $\mathrm{M}$ is then given upon integration of Eq.2 for $\theta$ ranging between $\pi$ and $\pi / 2$.

$$
\begin{aligned}
\mathbf{E}_{\mathrm{rad}}^{\mathrm{elbow}}(M, t) & =\frac{1}{4 \pi \epsilon_{0}} \frac{\tilde{J}_{t}}{\omega} e^{-j \omega t} R_{w} \\
& \times\left\{\begin{array}{l}
\int_{\pi}^{\pi / 2} \frac{3(y \cos \theta-x \sin \theta) \times\left(x-R_{w} \cos \theta\right)}{r_{\theta}^{5}} d \theta-\int_{\pi}^{\pi / 2} \\
\int_{\pi}^{\pi / 2} \frac{3(y \cos \theta-x \sin \theta) \times\left(y-R_{w} \sin \theta\right)}{r_{\theta}^{5}} d \theta-\int_{\pi}^{\pi / 2} \frac{t}{r_{\theta}^{5}} d \\
\int_{\pi}^{\pi / 2} \frac{3(y \cos \theta-x \sin \theta) \times z}{r} d \theta
\end{array}\right.
\end{aligned}
$$

where $r_{\theta}=\sqrt{\left(x-R_{w} \cos \theta\right)^{2}+\left(y-R_{w} \sin \theta\right)^{2}+z^{2}}$. Equation 5 is exact for any observation point. Nevertheless, at large distance from the elbowed area, $r \gg R_{w}$, the equation simplifies to:

$$
\mathbf{E}_{\mathrm{rad}}^{\mathrm{elbow}}(M, t)=\frac{1}{4 \pi \epsilon_{0}} \frac{j \widetilde{I}_{t}}{\omega} e^{-j \omega t} R_{w}\left\{\frac{3 \mathbf{r} \cdot \mathbf{u}}{r^{5}} \mathbf{r}-\frac{\mathbf{u}}{r^{3}}\right\},
$$

where $\mathbf{u}=\mathbf{e}_{\mathbf{x}}+\mathbf{e}_{\mathbf{y}}$. Thus, Eq.6 states that the current flowing in the quarter of circle radiates an EM field whose structure is similar to the one radiated by a dipole $\mathbf{p}_{\mathbf{q c}}=$ $p_{q c} \mathbf{u}$, with $p_{q c}=\sqrt{2} \widetilde{I}_{t} R_{w} / \omega$, located in $O$ and oriented at $\pi / 4$ w.r.t the $x$-axis (cf. fig. $6 \mathrm{~d}$ ).

\section{Total EM radiated field}

In summary, the total EM radiated field can be estimated by superposition of: (i)-Eq.4 applied to segments $A^{\prime} A, B C$ and $B^{\prime} C^{\prime}$; (ii)-Eq.6 applied to curves $A B$ and $A^{\prime} B^{\prime}$ assuming $r \gg R_{w}$ (dipolar contribution).

Due to the distance dependence of the former equations and to the fact that $L \gg W$, the major part of the EM radiated field able to influence the QTF can thus be restricted to the contribution of two point charges located in $A$ and $B$, plus the dipolar contribution of the quarter of circle $A B$. Along that line, if one assumes that at time $t$, the current flows along $A B$ curve from $A$ - to $B$-point (cf. elementary dipole orientation $\mathbf{d p}$ in figs.6a- and e), then Eq.4 tells us that $A$-point is positively charged and $B$-point is negatively charged. For $r \gg R_{w}$, the contribution of the two point charges reduces to a dipolar contribution as well, whose dipole $\mathbf{p}_{\mathrm{seg}}$, according to the conventions defined above, is oriented from $B-$ to $A$-point (cf. fig.6e).

$\mathbf{p}_{\text {seg }}$ and $\mathbf{p}_{\mathrm{qc}}$ have similar magnitudes: $j \widetilde{I}(t) e^{-j \omega t} / \omega$. They are parallel, $R_{w}$-far apart, but with opposite directions. This ultimately depicts a quadrupolar EM field. The magnitude of an EM field issued from a quadrupole estimated at a distance $r$, large enough from its center, is ${ }^{39}$ :

$$
E_{\mathrm{rad}}(\mathbf{r}, t) \simeq \frac{1}{4 \pi \epsilon_{0}} \frac{R_{w}^{2}}{\mathbf{r}^{4}} \frac{j \widetilde{I}(t)}{\omega} e^{-j \omega t}
$$

The above equation describes the radiated EM field for the case of an oversimplified geometry of the tip wire. With actual probes, one expects that geometry to be more complex and, hence, the structure of the resulting field too. This might explain why between distinct probes, the detailed geometrical configuration of the tip be $\frac{\sin \theta}{r_{\theta}}$ deffferent (gluing conditions, read-out wire geometry ${ }^{\theta} . d \theta$ the coupling may differ significantly.

$r$ We finally discuss the structure of the current source. Equation 7 stands for a pure harmonic source. In the generic case of a periodic current $\widetilde{I}(t)$ (pulsation $\left.\omega_{0}\right)$, its 
Fourier expansion must be considered instead: $\widetilde{I}_{t}(t)=$ $\left\langle\widetilde{I}_{t}\right\rangle+\sum_{n \neq 0} I_{n} e^{j \omega_{n} t}, \omega_{n}=n \omega_{0}, I_{n}$ and $\left\langle I_{t}\right\rangle$ being the $\mathrm{n}^{\text {th }}$ harmonic pulsation $(n \neq 0)$, the magnitude of the $\mathrm{n}^{\text {th }}$ Fourier component $(n \neq 0)$ and the static component $(n=0)$ of the current, respectively. Thus, the radiated field becomes:

$$
E_{\mathrm{rad}}(\mathbf{r}, t) \simeq \frac{1}{4 \pi \epsilon_{0}} \frac{R_{w}^{2}}{\mathbf{r}^{4}} \sum_{n=-\infty, \neq 0}^{+\infty} \frac{j I_{n}}{\omega_{n}} e^{-j \omega_{n} t}
$$

In Eq.8, the term under summation does not consider the static component as it does not promote the radiated field. This equation can thus be written in an integral form as follows:

$$
E_{\mathrm{rad}}(\mathbf{r}, t) \simeq \frac{1}{4 \pi \epsilon_{0}} \frac{R_{w}^{2}}{\mathbf{r}^{4}} \int_{0}^{t}\left[\widetilde{I}_{t}\left(t^{\prime}\right)-\left\langle\widetilde{I}_{t}\right\rangle\right] d t^{\prime},
$$

where the integrand stands for the AC part of the current source, as expected for the EM radiation source in standard antenna theory.

\section{B. Coupling to the QTF}

The derivation of the strain field within the QTF induced by the EM radiated field (Eq.9) is a key issue for the description of the coupling. Nevertheless, an analytical approach seems illusive because, although the calculation of the EM radiated field is oversimplified, it yet yields a non-linear distance dependence which makes the detailed tensorial calculations of the piezoelectric coupling hardly tractable. We therefore chose to discuss the coupling semi-quantitatively.

The quadrupolar contribution of the EM radiated field may be quantified by introducing two characteristic lengthes: $L_{c}$ and $R_{w}, R_{w}$ being the radius of curvature of the elbowed area of the wire yet defined. $L_{c}$ depicts the size of the influence zone of the EM field where the maximum coupling with the QTF is expected to occur (figs.6a and $\mathrm{b}$, dotted area). We assume $L_{c}$ to match the QTF prong's width: $L_{c} \simeq W \simeq 100 \mu \mathrm{m}$ and $R_{w} \simeq 50 \mu \mathrm{m}$ to match commonly reported values with QTF-based SPM probes $^{26,33}$.

In the context of nc-AFM/STM combined experiments, the tunnelling current exhibits an exponentiallike dependence with the tip-surface separation. Following the discussion at the end of subsection III A 3, the dynamic tunneling current $\widetilde{I}_{t}(t)$ stemming from the tip oscillatory motion exhibits $\mathrm{AC}, \widetilde{I}_{t}$, and static, $\left\langle\widetilde{I}_{t}\right\rangle$, components. $\left\langle\widetilde{I}_{t}\right\rangle$ stands for the experimentally reported observable (1 pA-1 nA range). In this context, Eq.9 writes:

$$
E_{\mathrm{rad}}\left(L_{c}, t\right) \simeq \frac{1}{4 \pi \epsilon_{0}} \frac{R_{w}^{2}}{L_{c}^{4}} \int_{0}^{t}\left[\widetilde{I}_{t}\left(t^{\prime}\right)-\left\langle\widetilde{I}_{t}\right\rangle\right] d t^{\prime}
$$

Fourier analysis states that $\widetilde{I}_{t}(t)$ is a superposition of harmonic waveforms with frequencies $f_{n}=n f_{0}(n \in \mathbb{N})$. For oscillation amplitudes $A_{0} \lesssim 1 \AA$, the first harmonics $(n \leq$ 3) preponderate with magnitudes $\simeq\left\langle\widetilde{I}_{t}\right\rangle$ (cf. appendix Aand ref.[26]). Moreover, the nc-AFM electronics behaves as a spectral filter that continuously tracks the resonance frequency $f_{0}$ of the QTF. Hence, the unique component of the $\widetilde{I}_{t}(t)$ Fourier expansion influencing the QTF is the fundamental one, whose magnitude is $\simeq\left\langle\widetilde{I}_{t}\right\rangle$. Therefore, the current writes: $\widetilde{I}_{t}(t)=\left\langle\widetilde{I}_{t}\right\rangle \cos \left(2 \pi f_{0} t\right)$, which is the waveform used so far, indeed. Thus:

$$
E_{\mathrm{rad}}\left(L_{c}, t\right) \simeq \frac{1}{4 \pi \epsilon_{0}} \frac{R_{w}^{2}}{L_{c}^{4}} \frac{\left\langle\widetilde{I}_{t}\right\rangle}{2 \pi f_{0}} \sin \left(2 \pi f_{0} t\right)
$$

Realistic experimental values $\left\langle\widetilde{I}_{t}\right\rangle=25 \mathrm{pA}$ and $f_{0} \simeq$ $32 \mathrm{kHz}$ yield $E_{\mathrm{rad}} \simeq 30 \mathrm{~V} / \mathrm{m}$. The corresponding mean radiated power per oscillation cycle in a volume $L_{c}^{3}$ at distance $L_{c}$ from the wire is $P_{\mathrm{rad}} \simeq \epsilon_{0} f_{0}\left\langle E_{\mathrm{rad}}^{2}\right\rangle L_{c}^{3} / 2 \simeq$ $810^{-20}\left\langle\widetilde{I}_{t}\right\rangle^{2} \mathrm{~W}$, if $\left\langle\widetilde{I}_{t}\right\rangle$ is in $\mathrm{pA}$. If the QTF is depicted by a $1 \mathrm{D}$ harmonic oscillator, the latter power can be compared to the one dissipated by friction during one cycle: $P_{\text {dis }}=f_{0} / 2 \times k / Q \times A_{0}^{2} \simeq 710^{-18} \times A_{0}^{2} \mathrm{~W}$, with $k / Q \simeq 1 / 25$ and $A_{0}$ in $\AA$. In the steady state, $P_{\text {dis }}$ equates the power supplied by external excitation to the QTF. For $P_{\text {dis }}=P_{\text {rad }}$, we find that $\left\langle\widetilde{I}_{t}\right\rangle \simeq 25 \mathrm{pA}$ may promote $\mathrm{a} \simeq 300$ pm-oscillation of the QTF!

Obviously, this result is overestimated owing to the $100 \%$ coupling efficiency we have assumed. A QTF converts electrical energy into mechanical energy by inverse piezoelectric effect, whose efficiency for quartz is weak. X-cut $\alpha$-quartz crystals have two non-equivalent piezoelectric tensor components ${ }^{40}\left(e_{11}, e_{14}\right)$ of magnitude $e \simeq 10^{-12} \mathrm{~m} / \mathrm{V}$. Therefore with $E_{\mathrm{rad}}=30 \mathrm{~V} / \mathrm{m}$, the resulting piezoelectric displacement is expected to be $u_{p} \simeq e E_{\mathrm{rad}} L_{c} \simeq 3 \mathrm{fm}$ only. However, the radiated energy has an oscillating character, and so has $u_{p}$. This dynamic displacement concerns a characteristic mass $\delta m=\rho L_{c}^{3}$ (for quartz, $\rho=210^{3} \mathrm{~kg} / \mathrm{m}^{3}$ ) located at the QTF prong's end, which acts as an oscillating inertial force of magnitude $F_{\text {inert }}=\delta m \partial^{2} u_{p} / \partial t^{2}=\left(2 \pi f_{0}\right)^{2} \delta m e E_{\mathrm{rad}} L_{c} \simeq$ $0.23 \mathrm{pN}$. If properly oriented, $F_{\text {inert }}(t)$ can excite the anti-symmetric mode of the QTF and trigger a mechanical oscillation of the prong with an amplitude $A_{\text {inert }}=$ $(Q / k) F_{\text {inert }} \simeq 6 \mathrm{pm}$, at $f_{0}$. That estimate is not negligible in view of the amplitude range used with QTF-based SPM probes, typically $A_{0} \simeq 40 \mathrm{pm}$ in the low-amplitude regime $^{2,3}$. The present effect, named inertial coupling, yields the bending of the QTF prong and, by virtue of the direct piezoelectric effect, a piezoelectric current component $I_{\text {inert }}\left(\widetilde{I}_{t}\right)$, which must scale linearly with $\widetilde{I}_{t}$. The total piezoelectric current $I_{p}\left(\widetilde{I}_{t}\right)$ supplied by the QTF to the nc-AFM electronics becomes an $\widetilde{I}_{t}$-dependent functional of the form: 


$$
I_{p}\left(\widetilde{I}_{t}\right)=I_{\mathrm{nf}}+\overbrace{I_{\mathrm{th}}+I_{\mathrm{osc}}+\underbrace{I_{\text {inert }}\left(\widetilde{I}_{t}\right)}_{\text {coupling }}+I_{\mathrm{ind}}\left(\widetilde{I}_{t}\right)}^{\text {mechanical motion }},
$$

where $I_{\mathrm{nf}}, I_{\mathrm{th}}$ and $I_{\mathrm{osc}}$ are the noise floor current (circuitry-dependent), the thermal noise current (weak, and essentially non-null at resonance) and the current produced by the QTF oscillation upon external excitation, respectively. In absence of mechanical, or electrical actuation, $I_{\mathrm{osc}}=0$, obviously. $I_{\mathrm{th}}, I_{\mathrm{osc}}$ and $I_{\text {inert }}\left(\widetilde{I}_{t}\right)$ stand for an actual mechanical oscillation of the QTF prong, but only $I_{\text {inert }}\left(\widetilde{I}_{t}\right)$ reflects a motion due to the $\widetilde{I}_{t}$-coupling.

In Eq.12, the last term $I_{\text {ind }}\left(\widetilde{I}_{t}\right)$ is also $\widetilde{I}_{t}$-dependent and reflects a current of induced charges due to the influence of the EM radiated field on the metallic electrodes of the QTF. These charges stem from (or flow to) the ground and load the electrodes with a density reflecting the inhomogeneous spatial distribution of $E_{\mathrm{rad}} \propto L_{c}^{-4}$ and the electrodes' geometry. This effect is named induction coupling and should scale linearly with $\widetilde{I}_{t}$ too. It cannot be excluded that $I_{\text {ind }}\left(\widetilde{I}_{t}\right)$ does partly contribute to the prong motion. If the induced charge distribution on the electrodes yields internal field lines with optimal symmetry 41 (as those observed upon AC electrical actuation of the QTF), a bending motion might be promoted. However, $\mathbf{E}_{\text {rad }}$ behaves as a quadrupolar field and does not clearly exhibit such a symmetry. Therefore, despite its AC character, $I_{\text {ind }}\left(\widetilde{I}_{t}\right)$ should not contribute to the prong motion, to a large extent. But being part of the overall detected current, its contribution to the measured oscillation amplitude of the tip, $A_{0}$, is not to be neglected, as discussed below and in subsection IIID.

\section{Electrical equivalence}

To help in interpreting the experimental spectra by means of the constitutive equation 12 , we reasoned in terms of electrical analogy of the QTF and its circuitry. The mostly reported ${ }^{42-46}$ electrical analogy of the QTF is the Butterworth-Van Dyke equivalent circuit, i.e. a RLC branch (impedance: $Z_{\mathrm{RLC}}(f)=R+j L 2 \pi f+1 /(j C 2 \pi f)$, with $\left.j^{2}=-1\right)$ in parallel with a capacitance $C_{L}$, both being electrically loaded by a common AC voltage supply $V_{\text {sup }}$ (cf. fig.7a). For ours, $C_{L}=12.5 \mathrm{pF}$ (manufacturer's data). The resonance/anti-resonance behavior of the QTF within this analogy is well-known and shown in fig.7b. The RLC branch depicts the current due to the mechanical motion of the QTF, whereas the $C_{L}$ branch depicts the capacitive charge that occurs in the electrodes due to $V_{\text {sup }}$.

The $I_{p}^{\mathrm{eq}}\left(f, \widetilde{I}_{t}\right)$ experimental spectrum (cf. fig.7d, reported from fig.5b for $\widetilde{I}_{t}=1.25 \mathrm{nA}$ ) has a very similar appearance as the one derived from the Butterworth-

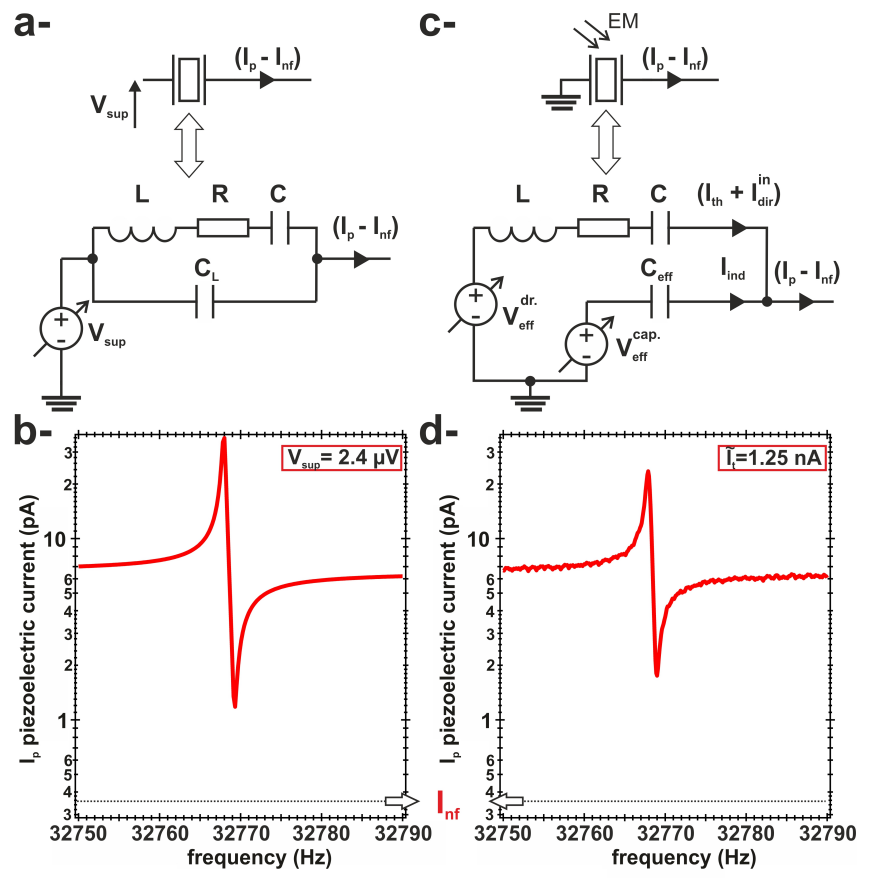

FIG. 7: (Color online) a-Butterworth-Van Dyke circuit used to describe the QTF when electrically actuated by an external supply $\left(V_{\text {sup }}\right)$. b-5Spice ${ }^{\complement}$ simulation of the $\mathrm{TF}$ of the Butterworth-Van Dyke circuit shown in a-. The $R, L$, $C$ parameters are those formerly derived for our QTF and the $\mathrm{I} / \mathrm{V}$ converter $\mathrm{TF}$ was included in the simulation. To achieve a background current of $\simeq 7 \mathrm{pA}$, consistently with the experimental spectrum shown in $\mathrm{d}$-, we need to input $V_{\text {sup }}=2.4 \mu \mathrm{V}$. The resonance/anti-resonance behavior is wellknown with this kind of circuit. c-Equivalent circuit of the QTF upon EM excitation. The Butterworth-Van Dyke circuit is reconsidered. The two branches are separated and excited by independent effective voltage sources. d-Experimental $I_{p}\left(f, \widetilde{I}_{t}\right)$ spectrum reported from fig. $5 \mathrm{~b}$ for $\widetilde{I}_{t}=1.25 \mathrm{nA}$. The resonance/anti-resonance behavior is qualitatively similar as the one shown in b-.

Van Dyke TF shown in fig.7b. The former circuit cannot be used to fit our data though, our setup including no explicit electrical actuation $\left(V_{\text {sup }}\right)$. Nevertheless, the above description remains partly correct. Indeed, $I_{\text {inert }}\left(\widetilde{I}_{t}\right)$ stems from a motion of the QTF prong which can be described as an effective voltage $V_{\text {eff }}^{\text {dr }}$ driving $Z_{\mathrm{RLC}}$. As to $I_{\text {ind }}\left(\widetilde{I}_{t}\right)$, it may be interpreted as an equivalent current resulting from an effective voltage $V_{\text {eff }}^{\text {cap }} \neq V_{\text {eff }}^{\text {dr }}$ loading an effective capacitance $C_{\text {eff. }} C_{\text {eff }} \neq C_{L}$ because $\mathbf{E}_{\text {rad }}$ does not load the electrodes as $V_{\text {sup }}$ would do. Thus, we ultimately propose in fig.7c a more generic equivalent circuit to the QTF whose piezoelectric current $I_{p}^{\mathrm{eq}}\left(f, \widetilde{I}_{t}\right)$ is: 
$I_{p}^{\mathrm{eq}}\left(f, \widetilde{I}_{t}\right)=I_{\mathrm{nf}}+I_{\mathrm{th}}+\overbrace{\frac{V_{\mathrm{eff}}^{\mathrm{dr}}\left(\widetilde{I}_{t}\right)}{Z_{\mathrm{RLC}}(f)}}^{I_{\mathrm{inert}}\left(f, \widetilde{I}_{t}\right)}+\overbrace{V_{\mathrm{eff}}^{\mathrm{cap}} C_{\mathrm{eff}}\left(\widetilde{I}_{t}\right) \times j(2 \pi f)}^{I_{\mathrm{ind}}\left(f, \widetilde{I}_{t}\right)}$,

Because $I_{\mathrm{nf}}, I_{\mathrm{th}}$ and the RLC parameters of the QTF are determined yet (cf. subsection IID), Eq.13 can be used as a two-parameters fitting functional: $V_{\text {eff }}^{\mathrm{dr}}\left(\widetilde{I}_{t}\right)$ and the $V_{\text {eff }}^{\text {cap }} C_{\text {eff }}\left(\widetilde{I}_{t}\right)$ product, both proportional to $\widetilde{I}_{t} \cdot I_{p}^{\text {eq }}\left(f, \widetilde{I}_{t}\right)$ being complex, the actual current used for fitting is $\left|I_{p}^{\text {eq }}\left(f, \widetilde{I}_{t}\right)\right|$, which properly treats the specific phase relationship between $I_{\text {inert }}\left(f, \widetilde{I}_{t}\right)$ and $I_{\text {ind }}\left(f, \widetilde{I}_{t}\right)$.

\section{Interpretation of the fits}

In fig.8a, the fits of the experimental spectra derived from Eq.13 have been overlaid (black curves) to the experimental spectra (coloured curves, reported from $5 \mathrm{~b}$ ). The as-derived fitting parameters are gathered in table I. The excellent agreement between the experimental and fitted curves over the entire frequency domain validate our paradigm. Eq.13 also permits the deconvolution of the total current for all frequencies, among which $f_{0}$. Thus, $\left|I_{p}^{\mathrm{eq}}\left(f_{0}, \widetilde{I}_{t}\right)\right|$ (green circles in fig.8b) may be deconvoluted as: (i)-the $\left|I_{\text {inert }}\left(f_{0}, \widetilde{I}_{t}\right)\right|$ contribution (red triangles in fig.8b) and (ii)-the $\left|I_{\text {ind }}\left(f_{0}, \widetilde{I}_{t}\right)\right|$ contribution (black squares in fig.8b). Figure $8 \mathrm{~b}$ shows the expected linearity between $\left|I_{p}^{\text {eq }}\left(f_{0}, \widetilde{I}_{t}\right)\right|,\left|I_{\text {inert }}\left(f_{0}, \widetilde{I}_{t}\right)\right|,\left|I_{\text {ind }}\left(f_{0}, \widetilde{I}_{t}\right)\right|$ and $\widetilde{I}_{t}$.

The modulii and arguments of the current components $\left(I_{p}^{\text {eq }}\left(f, \widetilde{I}_{t}\right)-I_{\mathrm{nf}}\right), I_{\text {inert }}\left(f, \widetilde{I}_{t}\right)$ and $I_{\text {ind }}\left(f, \widetilde{I}_{t}\right)$ are reported in fig.9a and b, respectively. For the sake of the clarity, we have reported them for $\widetilde{I}_{t}=1250,750,500$ and $250 \mathrm{pA}$ in fig.9a and for $\widetilde{I}_{t}=1250 \mathrm{pA}$ only in fig.9b (similar behavior otherwise). It can be seen that the induction current acts as a nearly constant background shift of the curve, whereas the inertial current is responsible for the resonant behavior at $f_{0}=32768 \mathrm{~Hz}$. However, it is the specific phase relationship between those components (inphase for $f<f_{0}, \pi / 2$-shifted for $f=f_{0}$ and $\pi$-shifted for $f>f_{0}$ ) which is responsible for the occurrence of the anti-resonance above $f_{0}(32769 \mathrm{~Hz})$. Another aspect of that peculiar phase relationship between components is the fact that the modulus of the equivalent current does not result from the direct summation of the modulii of its constitutive parts (cf. table I).

At last, it is useful to convert those currents into oscillation amplitude in order to assess their relative weight compared to typical oscillation amplitudes reported with QTF-based SPM probes, $A_{0} \simeq 40 \mathrm{pm}$. For that purpose, we chose to use the conversion factor $\gamma=0.505 \mathrm{~m} / \mathrm{A}$ given for conventional QTF by R.Grober et al. ${ }^{38}$. The conversion is shown on the right-axis of fig.8b. The inertial component $I_{\text {inert }}\left(f_{0}\right)$

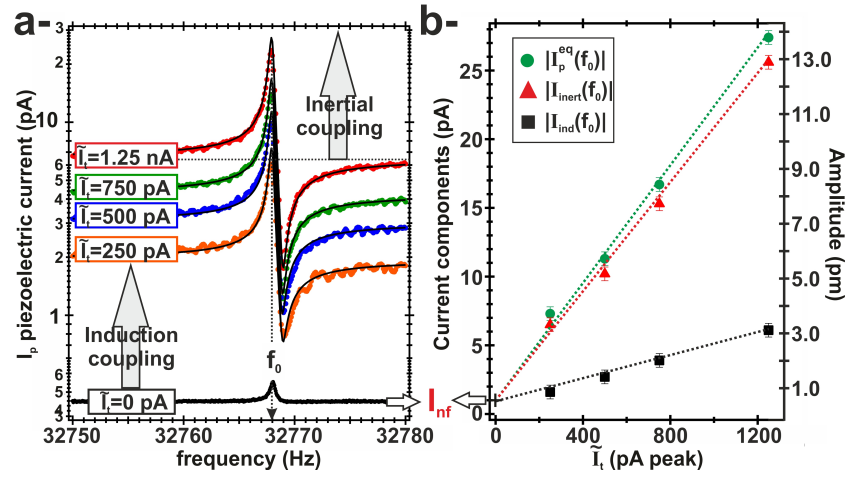

FIG. 8: (Color online). a- $I_{p}(f)$ spectra (log scale) for various $\widetilde{I}_{t}$ currents reported from fig.5b. The experimental spectra are fitted with $\left|I_{p}^{\mathrm{eq}}\left(f, \widetilde{I}_{t}\right)\right|$ (black curves, Eq.13) derived from the QTF equivalent circuit (fig.7c), which permits to assign the resonance/anti-resonance behavior $\left(\widetilde{I}_{t} \neq 0\right)$ to inertial/induction currents due to the EM radiation field on the QTF. b- $\left|I_{p}^{\text {eq }}\left(f_{0}\right)\right|,\left|I_{\text {inert }}\left(f_{0}\right)\right|$ and $\left|I_{\text {ind }}\left(f_{0}\right)\right|$ values derived from the fits (left scale, error bars $\pm 0.5 \mathrm{pA}$ ) vs. $\widetilde{I}_{t}$ and corresponding amplitude (right scale, see text).

\begin{tabular}{ccccc}
\hline \hline$\widetilde{I}_{t}$ & $(\mathrm{pA} \mathrm{peak})$ & 1250 & 750 & 500 \\
\hline$V_{\text {eff }}^{\text {dr }}$ & $(\mu \mathrm{V})$ & 1.80 & 1.08 & 0.72 \\
$V_{\text {eff }}^{\text {cap }} C_{\text {eff }}$ & $\left(10^{-17} \mathrm{C}\right)$ & 2.99 & 1.89 & 1.30 \\
\hline$\left|I_{p}^{\text {eq }}\left(f_{0}\right)\right|$ & $(\mathrm{pA})$ & $(27.4 \pm 0.5)$ & $(16.7 \pm 0.5)$ & $(11.3 \pm 0.5)$ \\
$A_{0}=\gamma\left|I_{p}^{\text {eq }}\left(f_{0}\right)\right|$ & $(\mathrm{pm})$ & $(13.8 \pm 0.2)$ & $(8.4 \pm 0.2)$ & $(5.7 \pm 0.2)$ \\
\hline$\left|I_{\text {inert }}\left(f_{0}\right)\right|$ & $(\mathrm{pA})$ & $(25.6 \pm 0.5)$ & $(15.3 \pm 0.5)$ & $(10.2 \pm 0.5)$ \\
$A_{0}^{\text {act }}=\gamma\left|I_{\text {inert }}\left(f_{0}\right)\right|$ & $(\mathrm{pm})$ & $(12.9 \pm 0.2)$ & $(7.7 \pm 0.2)$ & $(5.2 \pm 0.2)$ \\
\hline$\left|I_{\text {ind }}\left(f_{0}\right)\right|$ & $(\mathrm{pA})$ & $(6.1 \pm 0.5)$ & $(3.9 \pm 0.5)$ & $(2.7 \pm 0.5)$ \\
$A_{0}^{\text {ind }}=\gamma\left|I_{\text {ind }}\left(f_{0}\right)\right|$ & $(\mathrm{pm})$ & $(3.1 \pm 0.2)$ & $(2.0 \pm 0.2)$ & $(1.4 \pm 0.2)$ \\
\hline$A_{0}^{\text {app }}=A_{0}-A_{0}^{\text {act }}$ & $(\mathrm{pm})$ & $(0.9 \pm 0.4)$ & $(0.7 \pm 0.4)$ & $(0.5 \pm 0.4)$ \\
\hline \hline
\end{tabular}

TABLE I: Table of the fits parameters and corresponding current components (modulii) and amplitude at resonance vs. $\widetilde{I}_{t}$.

(red triangles in fig.8b) relates to an actual prong oscillation of amplitude $A_{0}^{\text {act }}=\gamma\left|I_{\text {inert }}\left(f_{0}\right)\right|$. Conversely, the induction component $\left|I_{\text {ind }}\left(f_{0}\right)\right|$ (black squares in fig.8b) does not reflect any prong oscillation. It is referred to as $A_{0}^{\text {ind }}=\gamma\left|I_{\text {ind }}\left(f_{0}\right)\right|$. The peculiar phase relationship between those currents is also responsible for the fact that the total amplitude $A_{0}$ derived from $I_{p}^{\mathrm{eq}}$ is not given by: $A_{0}=\gamma\left[\left|I_{\text {inert }}\left(f_{0}\right)\right|+\left|I_{\text {ind }}\left(f_{0}\right)\right|\right]=$ $\left(A_{0}^{\text {act }}+A_{0}^{\text {ind }}\right)$, but by: $A_{0}=\gamma \times\left|I_{p}^{\mathrm{eq}}\left(f_{0}\right)\right|=$ $\gamma \sqrt{\Re\left\{I_{\text {nf }}+I_{\text {th }}+I_{\text {inert }}\left(f_{0}\right)\right\}^{2}+\Im\left\{I_{\text {inert }}\left(f_{0}\right)+I_{\text {ind }}\left(f_{0}\right)\right\}^{2}} \simeq$

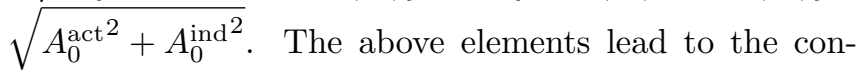
clusion that there is a gap between the actual oscillation amplitude of the QTF, $A_{0}^{\text {act }}$, and the detected one, simply referred to as $A_{0}$, so far. This gap will be referred to as an apparent amplitude $A_{0}^{\text {app }}$, also in given in table I: $A_{0}^{\text {app }}=A_{0}-A_{0}^{\text {act }}$. 


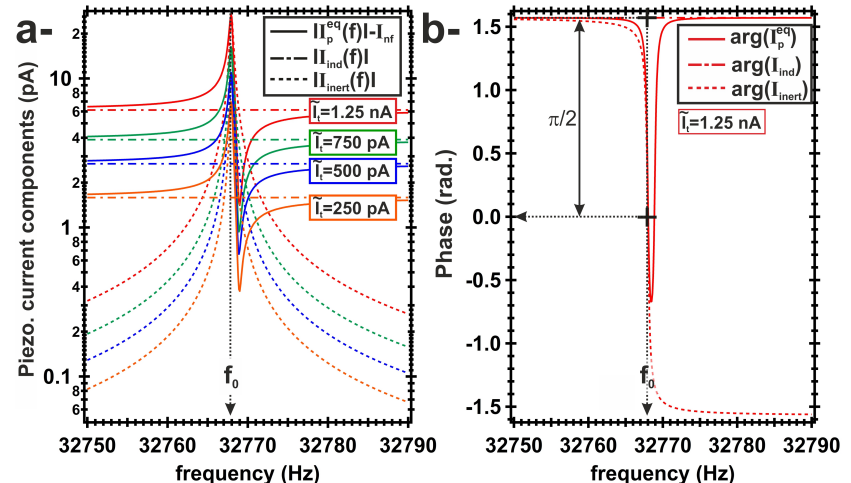

FIG. 9: (Color online). a-Current components after Eq.13 (log scale) vs. frequency for various $\widetilde{I}_{t}$ currents. The parameters used to compute the curves are those issued from the fits of the experimental spectra (Table I). One has reported: $\left(\left|I_{p}^{\mathrm{eq}}(f)\right|-I_{\mathrm{nf}}\right)$ (continuous colored lines), $\left|I_{\text {ind }}(f)\right|$ (double dotted colored lines) and $I_{\text {inert }}(f)$ (dotted colored lines). The inertial component is responsible for the resonance behavior and the induction component acts as constant background which shifts the resulting curve upwards. It is the peculiar phase relationship between both components (see b-), which is responsible for the anti-resonance behavior of the resulting total current. b- Corresponding arguments of the current components (radians) vs. frequency. For the sake of the clarity, we only have reported them for $\widetilde{I}_{t}=1.25 \mathrm{nA}$. It is visible that inertial and induction components are on-phase for $f<f_{0}, \pi / 2$ phase-shifted at resonance and anti-phased for $f>f_{0}$.

\section{CONSEQUENCES OF THE COUPLING IN NC-AFM: FREQUENCY SHIFT, OSCILLATION AMPLITUDE AND DAMPING}

The way $A_{0}^{\text {act }}$ and $A_{0}^{\text {app }}$ amplitudes (cf. section IIID) affect the experimental nc-AFM observables $(\Delta f$ and damping) is not trivial because one might expect the amplitude controller to compensate for them. In order to tackle this issue, we suggest the following thought experiment. Let us consider that the tip oscillates with an amplitude setpoint $A_{0}^{\text {set }}$ at a constant rest distance to the surface (few $\AA$ ). The amplitude controller is engaged (damping signal recorded), whereas the distance controller not. Let us now compare two situations, one with $\widetilde{I}_{t}=0$, the other one where $\widetilde{I}_{t} \neq 0$. Compared to the $\widetilde{I}_{t}=0$ case, $\widetilde{I}_{t} \neq 0$ leads to: (i)-an actual supplementary oscillation of the tip, $A_{0}^{\text {act }}$. However, $A_{0}^{\text {act }}$ is properly compensated by the controller, which brings the oscillation down to $A_{0}^{\text {set }}$; (ii)-an apparent amplitude $A_{0}^{\text {app }}$ which is also compensated by the controller, albeit not standing for a physical oscillation. The order of magnitude for these amplitudes may conditionally yield $10 \mathrm{pm}$ (cf. table I), that is $\simeq 25 \%$ of $A_{0}=40 \mathrm{pm}$. This should readily be visible in the damping signal, whose interpretation becomes delicate then.

Moreover, the actual oscillation amplitude of the tip and the one expected from $A_{0}^{\text {set }}$ are systematically shifted by $A_{0}^{\text {app }}$. This induces a shift of the tip/surface lower turning point separation, and hence, a difference between measured $\Delta f$ in both experiments.

This effect can be investigated in more detail. For this purpose, the influence that any $A_{0}$ change has on $\Delta f$ is to be derived. For that purpose, we expand Giessibl's formula ${ }^{27}$ linking $\Delta f$, the tip-sample force $F_{\mathrm{ts}}(z)$ and $A_{0}$ to obtain (cf. appendix B-):

$$
\frac{d \Delta f}{d A_{0}}=-\frac{A_{0} f_{0}}{8 k} \frac{\partial^{3} F_{\mathrm{ts}}(z)}{\partial z^{3}}
$$

With $f_{0}=32678 \mathrm{~Hz}, Q=50000$ and $k=1800 \mathrm{~N} / \mathrm{m}$ and a typical force magnitude of $\simeq-2 \mathrm{nN}$ in the $\mathrm{SR}$ attractive regime $\left(\simeq 3.5 \AA\right.$ to the surface, $\left.\left\langle\widetilde{I}_{t}\right\rangle \simeq 1 \mathrm{nA}\right)$ and $A_{0}=40 \mathrm{pm}$, any $A_{0}$ change as small as $d A_{0}=A_{0}^{\text {app }}=$ $1 \mathrm{pm}$ (cf. table I) yields: $d \Delta f \simeq 0.25 \mathrm{~Hz}$ (cf. fig.A.2 in appendix B-). This $d \Delta f$ change has a similar magnitude as some of the experimentally reported molecular corrugations upon constant height imaging (e.g. fig.2B in ref.[3]). Consequently, if quantitative analysis is targeted (force extraction by inversion algorithms ${ }^{47} \ldots$ ), the coupling reported here must be considered.

\section{CONCLUSION}

In this work, we have developed an experimental "proof of concept" setup aiming at exploring an intrinsic origin to the coupling between the frequency shift and the tunneling current, as reported with QTFbased SPM probes during nc-AFM/STM combined experiments. Our setup has allowed us to unambiguously show that an oscillating current flowing in a wire located in the direct vicinity of one of the QTF's prongs induces a flowing current between the electrodes of this QTF. An interpretation framework has been proposed, which captures the physics at play and details the response of the QTF. The oscillating current in the wire makes it behave like a tiny antenna which radiates an oscillating electromagnetic field whose frequency matches the QTF resonance frequency and hence, makes the rest of the process extremely efficient. This field penetrates the body of the QTF and, by virtue of the inverse piezoelectric effect, accelerates a volume of quartz located at the QTF prong's end. This accelerated piece of matter acts as an external, unexpected, actuation of the QTF and provokes its oscillation, which leads to a part of current, the so-called inertial current, collected by the QTF electrodes. The electromagnetic field also bathes the metallic electrodes, which by induction leads to another part of the detected current in the QTF. The induction-related current does not reflect any motion of the QTF prong. But, being part of the global current flowing in the electrodes, it might wrongly be interpreted as if it resulted from such a motion.

Inertial and induction currents get added to the overall piezoelectric current supplied by the QTF and trans- 
late into an actual supplementary oscillation amplitude and an apparent oscillation, respectively. These parasitic components ultimately fake the oscillation amplitude of the tip detected by the nc-AFM electronics, which alters both, the $\Delta f$ and the damping $(\Gamma)$ measurements, hence the $(\Delta f, \Gamma) / I_{t}$ coupling.

The aim of our work was to qualitatively evince the coupling between an EM field and a QTF covered with electrodes. The geometry of the radiating tunneling wire was chosen according to the available literature for both, experiments and calculations. Nevertheless, we think that with the chosen geometry, the effect measured in our experimental setup with respect to generally used qPlus sensors is rather underestimated. Indeed, for the short decay length of the EM radiated field as described in our work, the distance between the tip wire and the QTF prong's end is crucial. In our experimental setup, the centre of the wire is almost one order of magnitude farther away than with commonly used tunneling wires $(50 \mu \mathrm{m}$ in diameter) which are directly glued at the QTF prong's end. While on the one hand, optimized tip lengths and bending radii could decrease the coupling effect, on the other hand, the large diameter of the wire and the large separation between the QTF front end and the wire in our setup (due to the fact that the wire is not glued onto the prong end) result in a measured effect which might be up to an order of magnitude smaller than with a thinner wire glued directly on the QTF.

The detailed implications the coupling might have on the quantitative interpretation of nc-AFM/STM data can only be assessed by means of a complete quantitative approach. Both, the electromagnetic and the coupled piezoelectric/mechanical problems must jointly be solved by finite element method (FEM) calculations. Once the magnitude of each effect (inertial, induction) is known, its influence could be assessed with our nc-AFM simulator, where the complete set of closed loops is reproduced in detail. Thus, the FEM/nc-AFM simulator combination might exactly quantify the influence the tunnelling current has on $\Delta f$ and damping signals. This work is currently in progress.

At last, we can suggest some exploratory solutions to avoid the coupling reported here. The most straightforward one consists in operating the QTF at low tunnelling currents, in the range of $10 \mathrm{pA}$. Another approach requires to modify or re-design the QTF. Two conditions have to be fulfilled then: (i)-the metallic electrodes need to be located as far as possible from the tip; (ii)-the piezoelectricity property of the QTF prong's end where the tip is glued needs to be destroyed. For that purpose, one might suggest to perform a well-controlled chemical etching of the electrodes and an amorphization of the prong's end (by Helium implantation for instance). This solution would preserve the quality factor of the QTF, but the implantation process might be delicate. A second option might consist in elongating the QTF prong's end by attaching to it a proper part made of a non piezoelectric material at the end of which the tip would be glued. This solution might be easier to implement, but the mechanical properties of the QTF, among which its quality factor, should be altered.

\section{Acknowledgments}

We thank F.Dérivaux and A.Bchini from the IM2NP for technical support, T.Léoni, C.Becker, H.Klein (Cinam, Marseille), J.-M.Themlin, S.Clair from the IM2NP, S.Gauthier (CEMES, Toulouse), R.Pawlak (Uni. Basel, Switzerland), A.Sweetman and M.Baykara for fruitful discussions and acknowledge support from the ANR NANOKAN program (ANR-11-BS100004). 

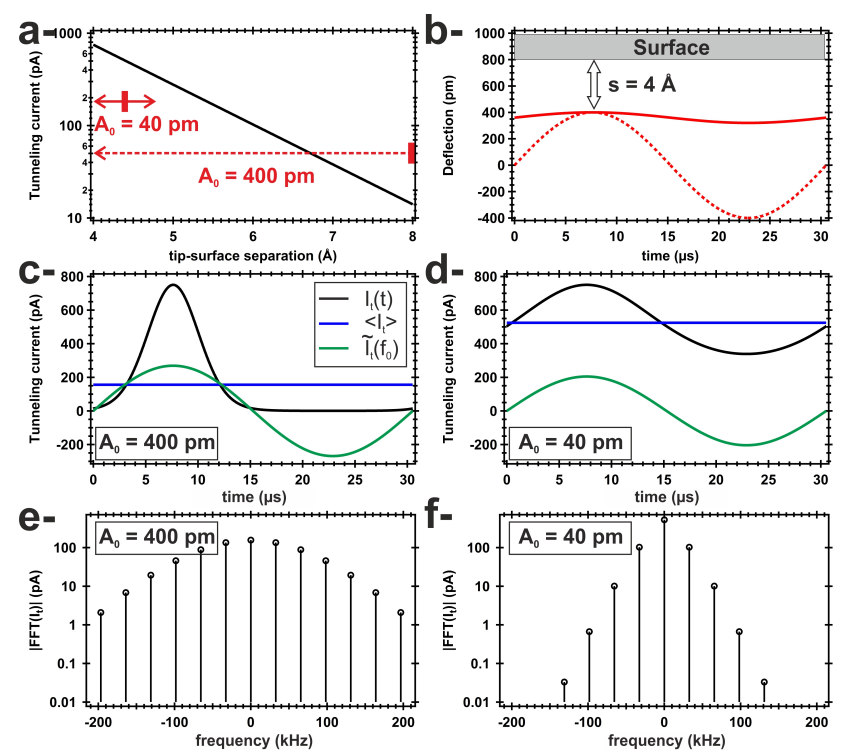

FIG. A.1: (Color online). a-Tunneling current (log scale) vs. tip-surface separation, after Eq.A.1, with $\lambda=1 \AA$ and $I_{0}=40 \mathrm{nA}$. The trace of the tip oscillation has been indicated for $A_{0}=40$ and $400 \mathrm{pm}$. b-Trace of the tip oscillation at $f_{0}=32768 \mathrm{~Hz}$ vs. time for $A_{0}=40 \mathrm{pm}$ (continuous curve) and $A_{0}=400 \mathrm{pm}$ (dotted curve). The $A_{0}=40 \mathrm{pm}$-curve has been arbitrarily shifted vertically. For both amplitudes, the tunneling current $\widetilde{I}_{t}(t)$ reported in c- and d- is calculated for a common lower turning point $s=z_{0}-A_{0}=4 \AA$. cTunneling current (black curve) vs. time for $A_{0}=400 \mathrm{pm}$. The blue and green curves depict the corresponding static $(n=0)$ and fundamental components $(n=1)$ as derived from the FFT spectrum shown in e-, respectively. d-Same as c- for $A_{0}=40 \mathrm{pm}$. e-FFT spectrum (modulus, log scale) of the tunneling current shown in c- (bilateral representation, phase components not shown). f-FFT spectrum of the tunneling current shown in d-.

\section{APPENDICES}

\section{A- Tunneling current Fourier components}

The simplified expression for the instantaneous tunnelling current $\widetilde{I}_{t}(t)$ flowing through the oscillating tip separated by $z(t)$ from the surface is written:

$$
\widetilde{I}_{t}(t)=I_{0} e^{-z(t) / \lambda},
$$

where $\lambda$ is a characteristic length which pertains to the work function of the substrate material. For metals, $\lambda \simeq 1 \AA$. Assuming a harmonic motion of the tip, the instantaneous tip-surface separation may be written as: $z(t)=z_{0}+A_{0} \cos \left(2 \pi f_{0} t\right)$, where $z_{0}$ is the tip-surface rest separation and $A_{0}$ the oscillation amplitude. Standard Fourier analysis allows for recasting $\widetilde{I}_{t}(t)$ as:

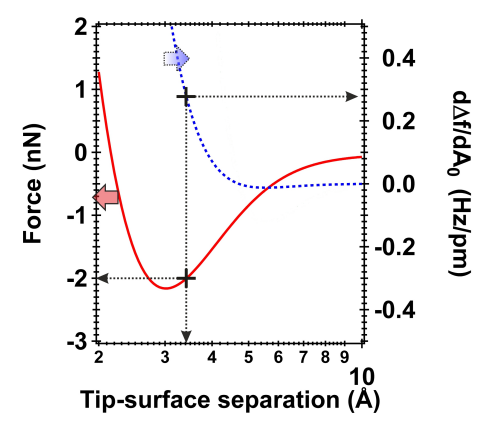

FIG. A.2: (Color online). Force (Eq.A.9, left scale) and $d \Delta f / d A_{0}$ (Eq.A.8, right scale) vs. tip-surface separation (log scale). One has reported the value of $d \Delta f / d A_{0}$ for $A_{0}=$ $40 \mathrm{pm}$ (blue dotted curve). We get $d \Delta f / d A_{0} \simeq 0.25 \mathrm{~Hz} / \mathrm{pm}$ at $z \simeq 3.5 \AA$ and a corresponding force $\simeq-2 \mathrm{nN}$.

$$
\begin{aligned}
\widetilde{I}_{t}(t) & =I_{0} e^{-z_{0} / \lambda}\left\{\text { BesselI }\left[0, \frac{A_{0}}{\lambda}\right]\right. \\
& \left.+2 \sum_{n=1}^{+\infty}(-1)^{n} \operatorname{BesselI}\left[n, \frac{A_{0}}{\lambda}\right] \cos \left(2 \pi n f_{0} t\right)\right\},
\end{aligned}
$$

where BesselI is the Bessel function of the first kind. Defining $\left\langle\widetilde{I}_{t}\right\rangle=I_{0} e^{-z_{0} / \lambda} \operatorname{BesselI}\left[0, A_{0} / \lambda\right]$ as the DC part of the tunnelling current, which also relates to the experimentally measured current, the AC part $\widetilde{I}_{t}(t)$ becomes:

$$
\widetilde{I}_{t}(t)=2\left\langle\widetilde{I}_{t}\right\rangle \sum_{n=1}^{+\infty}(-1)^{n} \frac{\operatorname{BesselI}\left[n, \frac{A_{0}}{\lambda}\right]}{\operatorname{BesselI}\left[0, \frac{A_{0}}{\lambda}\right]} \cos \left(2 \pi n f_{0} t\right)
$$

For $A_{0} / \lambda \simeq 1$, the amplitude of the first harmonics are around $\left\langle\widetilde{I}_{t}\right\rangle$, whereas higher ones decrease rapidly (cf. figs.A.1e- and $\mathrm{f}-$ ). We have shown the AC part is of the same order as the DC one for oscillation around $1 \AA$. The radiated EM field is also given by a Fourier summation, so only the knowledge of the contribution given by the $\mathrm{AC}$ part of the current is necessary.

\section{B- Relation between $d \Delta f$ and $d A_{0}$}

We here establish the relation between the $\Delta f$ change, $d \Delta f$, due to a $d A_{0}$ uncertainty (or bad estimate) of the actual oscillation amplitude of the tip, in the limit of the small-amplitude regime $\left(A_{0} \ll z_{0}\right.$, see hereafter $)$. The starting point is F. Giessibl's formula giving $\Delta f$ for a tipsurface interaction $F_{\text {ts }}$, a rest distance $z_{0}$, an amplitude $A_{0}$, a spring constant $k$ and a resonance frequency $f_{0}$, namely ${ }^{27}$ :

$$
\frac{\Delta f}{f_{0}}=-\frac{1}{2 k A_{0}} \int_{0}^{2 \pi} F_{\mathrm{ts}}\left(z_{0}+A_{0} \cos (x)\right) \cos (x) d x
$$

We calculate $\left(d \Delta f / f_{0}\right) / d A_{0}$ by restricting the expansion 
of the former equation w.r.t. to $A_{0}$ around $z_{0}$ to the lowest ordered non-zero term.

$$
\begin{aligned}
\frac{\frac{d \Delta f}{f_{0}}}{d A_{0}} & =-\frac{1}{2 k A_{0}}\left\{\left.\int_{0}^{2 \pi} \frac{\partial F_{\mathrm{ts}}}{\partial z}\right|_{z_{0}+A_{0} \cos (x)} \cos ^{2}(x) d x\right. \\
& \left.-\frac{1}{A_{0}} \int_{0}^{2 \pi} F_{\mathrm{ts}}\left(z_{0}+A_{0} \cos (x)\right) \cos (x) d x\right\}
\end{aligned}
$$

We expand the integrands as:

$$
\begin{aligned}
\left.\frac{\partial F_{\mathrm{ts}}}{\partial z}\right|_{z_{0}+A_{0} \cos (x)} & =\left.\frac{\partial F_{\mathrm{ts}}}{\partial z}\right|_{z_{0}}+\left.\frac{\partial^{2} F_{\mathrm{ts}}}{\partial z^{2}}\right|_{z_{0}} A_{0} \cos (x) \\
& +\left.\frac{1}{2} \frac{\partial^{3} F_{\mathrm{ts}}}{\partial z^{3}}\right|_{z_{0}} A_{0}^{2} \cos ^{2}(x)+o\left(A_{0}^{3} \cos ^{3}(x)\right)
\end{aligned}
$$

and:

$$
\begin{aligned}
F_{\mathrm{ts}}\left(z_{0}+A_{0} \cos (x)\right) & =F_{\mathrm{ts}}\left(z_{0}\right)+\left.\frac{\partial F_{\mathrm{ts}}}{\partial z}\right|_{z_{0}} A_{0} \cos (x) \\
& +\left.\frac{1}{2} \frac{\partial^{2} F_{\mathrm{ts}}}{\partial z^{2}}\right|_{z_{0}} A_{0}^{2} \cos ^{2}(x) \\
& +\left.\frac{1}{6} \frac{\partial^{3} F_{\mathrm{ts}}}{\partial z^{3}}\right|_{z_{0}} A_{0}^{3} \cos ^{3}(x)+o\left(A_{0}^{4} \cos ^{4}(x)\right)
\end{aligned}
$$

The latter developments are inserted into Eq.A.5 and we note that $\int_{0}^{2 \pi} \cos ^{n}(x) d x=0$ when $n$ is odd. Therefore, in the limit $A_{0} \ll z_{0}$, we ultimately get:

$$
\frac{d \Delta f}{d A_{0}}=-\left.\frac{A_{0} f_{0}}{8 k} \frac{\partial^{3} F_{\mathrm{ts}}}{\partial z^{3}}\right|_{z_{0}}
$$

The former equation has been plotted in fig.A.2 for $A_{0}=$ $40 \mathrm{pm}$. The other parameters are $f_{0}=32768 \mathrm{~Hz}$ and $k=1800 \mathrm{~N} / \mathrm{m}$. The tip-surface interaction force derives from a long-range Hamaker potential and a short-range Morse potential of kind:

$$
\begin{aligned}
F_{\mathrm{ts}}(z) & =-\frac{\partial V_{\mathrm{ts}}(z)}{\partial z} \\
& =-\frac{\partial}{\partial z}\left\{-\frac{H R}{6 z}-U_{0}\left[2 e^{-\left(z-r_{c}\right) / \lambda}-e^{-2\left(z-r_{c}\right) / \lambda}\right]\right\}
\end{aligned}
$$

where $R$ stands for the tip radius. We chose $R=2 \mathrm{~nm}$. The other parameters have been chosen for a siliconsilicon interaction ${ }^{48}$, namely: $H=1.86510^{-19} \mathrm{~J}, U_{0}=$ $3.64110^{-19} \mathrm{~J}, \lambda=1.2 \AA$ and $r_{c}=2.357 \AA$.

For $A_{0}=40 \mathrm{pm}$, we get $d \Delta f / d A_{0} \simeq 0.25 \mathrm{~Hz} / \mathrm{pm}$ at $z \simeq 3.5 \AA$ and a corresponding force $\simeq-2 \mathrm{nN}$. Hence, for $d A_{0}=1 \mathrm{pm}, d \Delta f \simeq 0.25 \mathrm{~Hz}$.

References

${ }^{1}$ F.J. Giessibl, Appl. Phys. Lett. 76, 1470 (2000).

${ }^{2}$ L. Gross, F. Mohn, P. Liljeroth, J. Repp, F.J. Giessibl, and G. Meyer, Science 324, 1428 (2009).

${ }^{3}$ L. Gross, F. Mohn, N. Moll, and G. Meyer, Science 325, 1110 (2009).

${ }^{4}$ L. Gross, F. Mohn, P. Liljeroth, J. Repp, F.J. Giessibl, and G. Meyer, Nature Chemistry 2, 821 (2010).

${ }^{5}$ F. Mohn, J. Repp, L. Gross, G. Meyer, M.S. Dyer, and M. Persson, Phys. Rev. Lett. 105, 266102 (2010).

${ }^{6}$ K. Saitoh, K. Hayashi, Y. Shibayama, and K. Shirahama, Phys. Rev. Lett. 105, 236103 (2010).

7 M. Ternes, C. González, C.P. Lutz, P. Hapala, F.J. Giessibl, P. Jelínek, and A.J. Heinrich, Phys. Rev. Lett. 106, 016802 (2011).

${ }^{8}$ L. Gross, F. Mohn, N. Moll, B. Schuler, A. Criado, E. Guitián, D. Peña, A. Gourdon, and G. Meyer, Science 337, 1326 (2012).

9 F. Mohn, L. Gross, N. Moll, and G. Meyer, Nature Nanotechnology 7(4), 227 (2012).

10 R. Pawlak, S. Kawai, S. Fremy, T. Glatzel, and E. Meyer, J. Phys.: Cond. Matt. 24, 084005 (2012).

11 D.G. de Oteyza, P. Gorman, Y.-C. Chen, S. Wickenburg, A. Riss, D.J. Mowbray, G. Etkin, Z. Pedramrazi, H.-Z. Tsai, A. Rubio, M.F. Crommie, and F.R. Fischer, Science 340, 1434 (2013).

12 R. Pawlak, T. Glatzel, V. Pichot, L. Schmidlin, S. Kawai, S. Fremy, D. Spitzer, and E. Meyer, Nano Lett. 13, 5803 (2013).

13 F.Pielmeier and F.J. Giessibl, Phys. Rev. Lett. 110,
$266101(2013)$

14 B. Schuler, W. Liu, A. Tkatchenko, N. Moll, G. Meyer, A. Mistry, D. Fox, and L. Gross, Phys. Rev. Lett. 111, 106103 (2013).

15 J. van der Lit, M.P. Boneschanscher, D. Vanmaekelbergh, M. Ijäs, A. Uppstu, M. Ervasti, A. Harju, P. Liljeroth, and I. Swart, Nature Communications 4, 2023 (2013).

16 J. Zhang, P. Chen, B. Yuan, W. Ji, Z. Cheng, and X. Qiu, Science 342, 611 (2013).

17 A. M. Sweetman, S.P. Jarvis, H. Sang, I. Lekkas, P. Rahe, Yu Wang, J. Wang, N.R. Champness, L. Kantorovich, and P. Moriarty, Nature Communications 5, 3931 (2014).

18 M. Schneiderbauer, M. Emmrich, A. Weymouth, and F.J. Giessibl, Phys. Rev. Lett. 112, 166102 (2014).

19 B. Schuler, S.-X. Liu, Y. Geng, S. Decurtins, G. Meyer, and L. Gross, Nano Lett. 14, 3342 (2014).

${ }^{20}$ F. Huber, S. Matencio, A.J. Weymouth, C. Ocal, E. Barrena, and F.J. Giessibl, Phys. Rev. Lett. 115, 066101 (2015).

21 C. Wagner, M.F.B. Green, P. Leinen, T. Deilmann, P. Krüger, M. Rohlfing, R. Temirov, and F. S. Tautz, Phys. Rev. Lett. 115, 026101 (2015).

${ }^{22}$ P. Hapala, M. Svec, O. Stetsovych, N.J. van der Heijden, M. Ondrácek, J. van der Lit, P. Mutombo, I. Swart, and P. Jelínek, Nature Communications 7, 11560 (2016).

${ }^{23}$ S. Kawai, A.S. Foster, T. Björkman, S. Nowakowska, J. Björk, F. Federici Canova, L.H. Gade, T.A. Jung, and E. Meyer, Nature Communications 7, 11559 (2016).

24 J. van der Lit, F. Di Cicco, P. Hapala, P. Jelínek, and I. 
Swart, Phys. Rev. Lett. 116, 096102 (2016).

25 A.J. Weymouth, T. Wutscher, J. Welker, T. Hofmann, and F.J. Giessibl, Phys. Rev. Lett. 106, 226801 (2011).

26 Z. Majzik, M. Setvin, A. Bettac, A. Feltz, V. Cháb, and P. Jelínek, Beilstein J. Nanotechnol. 3, 249 (2012).

27 F.J. Giessibl, Phys. Rev. B 56, 16010 (1997).

28 The coupling in question has sometimes been referred to as a cross-talk, which relates to instrumental aspects. We will use the otherwise more generic term of coupling.

29 Non-contact Atomic Force Microscopy Vol.3, edited by S. Morita, F. Giessibl, E. Meyer, and R. Wiesendanger (Spinger, BERLIN, 2014).

30 T. Wutscher, A. Weymouth, and F.J. Giessibl, Phys. Rev. B 85, 195426 (2012).

31 M.Z. Baykara, M. Todorovic, H. Mönig, T.C. Schwendemann, L. Rodrigo, E.I. Altman, R. Pérez, and U.D. Schwarz, J. Phys. Chem. C 119, 6670 (2015).

32 Non-contact Atomic Force Microscopy Vol.2, edited by S. Morita, F. Giessibl, and R. Wiesendanger (Spinger, BERLIN, 2009).

33 J. Berger, M. Svec, M. Müller, M. Ledinsky, A. Fejfar, P. Jelínek, and Z. Majzik, Beilstein J. Nanotechnol. 4, 1 (2013).

34 M. Heyde, G. Simon, H.-P. Rust, and H.-J. Freund, Appl. Phys. Lett. 89, 263107 (2006).

35 http://www.ti.com/product/opa637.

36 F.J. Giessibl, F. Pielmeier, T. Eguchi, T. An, and Y. Hasegawa, Phys. Rev. B 84, 125409 (2011).
37 J. Lübbe, M. Temmen, P. Rahe, A. Kühnle, and M. Reichling, Beilstein J. Nanotechnol. 4, 227 (2013).

38 R.D. Grober, J. Acimovic, J. Schuck, D. Hessman, P.J. Kindlemann, J. Hespanha, A.S. Morse, K. Karrai, I. Tiemann, and S. Manus, Rev. Sci. Instrum. 71, 2776 (2000).

39 J. A. Stratton, Théorie de l'électromagnétsime (Dunod, Paris, 1961).

40 J. Yang, An introduction to the theory of piezoelectricity (Springer, BERLIN, 2005).

${ }^{41} \mathrm{~K}$. Karrai, Lecture notes on shear and friction force detection with quartz tuning forks, Work presented at the "Ecole thématique du CNRS" on near-field optics, 2000, la Londe les Maures, France.

42 J.-M. Friedt and E. Carry, Am. J. Phys. 75, 415 (2007).

${ }^{43}$ Y. Qin and R. Reifenberger, Review of Scientific Instruments 78, 063704 (2007).

${ }^{44}$ R. Oria, J. Otero, L. Gonzalez, L. Botaya, M. Carmona, and M. Puig-Vidal, Sensors 13, 7156 (2013).

${ }^{45}$ S. An, K. Lee, B. Kim, J. Kim, S. Kwon, Q. Kim, M. Lee, and W. Jhe, Current Applied Physics 13, 1899 (2013).

46 Nanonis, Technical note: "Piezoelectric Quartz Tuning Forks for Scanning Probe Microscopy", 2005, http://www.specs-zurich.com/upload/cms/user/TF2.pdf. 47 J. Sader and S. Jarvis, Appl. Phys. Lett. 84, 1801 (2004).

48 R. Pérez, I. Stich, M. Payne, and K. Terakura, Phys. Rev. B 58, 10835 (1998). 